\title{
Le potentiel d'innovation et de transformation de l'économie sociale: quelques éléments de problématique
}

\author{
Benoît Lévesque*
}

\section{Présentation du crises}

Notre Centre de recherche sur les innovations sociales (CRISES) est une organisation interuniversitaire qui étudie et analyse principalement «les innovations et les transformations sociales».

Une innovation sociale est une intervention initiée par des acteurs sociaux pour répondre à une aspiration, subvenir à un besoin, apporter une solution ou profiter d'une opportunité d'action afin de modifier des relations sociales, de transformer un cadre d'action ou de proposer de nouvelles orientations culturelles.

En se combinant, les innovations peuvent avoir à long terme une efficacité sociale qui dépasse le cadre du projet initial (entreprises, associations, etc.) et représenter un enjeu qui questionne les grands équilibres sociétaux. Elles deviennent alors une source de transformations sociales et peuvent contribuer à l'émergence de nouveaux modèles de développement.

Les chercheurs du CRISES étudient les innovations sociales à partir de trois axes complémentaires : développement et territoire, les conditions de vie et le travail et l'emploi.

\section{Axe innovations sociales, développement et territoire}

- Les membres de l'axe développement et territoire s'intéressent à la régulation, aux arrangements organisationnels et institutionnels, aux pratiques et stratégies d'acteurs socioéconomiques qui ont une conséquence sur le développement des collectivités et des territoires. Ils étudient les entreprises et les organisations (privées, publiques, coopératives et associatives) ainsi que leurs interrelations, les réseaux d'acteurs, les systèmes d'innovation, les modalités de gouvernance et les stratégies qui contribuent au développement durable des collectivités et des territoires.

\section{Axe innovations sociales et conditions de vie}

- Les membres de l'axe conditions de vie repèrent et analysent des innovations sociales visant l'amélioration des conditions de vie, notamment en ce qui concerne la consommation, l'emploi du temps, l'environnement familial, l'insertion sur le marché du travail, l'habitat, les revenus, la santé et la sécurité des personnes. Ces innovations se situent, généralement, à la jonction des politiques publiques et des mouvements sociaux : services collectifs, pratiques de résistance, luttes populaires, nouvelles manières de produire et de consommer, etc.

\section{Axes innovations sociales, travail et emploi}

- Les membres de l'axe travail et emploi orientent leurs recherches vers l'organisation du travail, la régulation de l'emploi et la gouvernance des entreprises dans le secteur manufacturier, dans les services, dans la fonction publique et dans l'économie du savoir.

\footnotetext{
* Est professeur titulaire associé à l'École nationale d'administration publique (ENAP) et à l'Université du Québec à Montréal (UQÀM), membre du Centre de recherche sur les innovations sociales (CRISES) et de l'Alliance de recherche universités-communautés en économie sociale (ARUC-ÉS).
} 
Les travaux portent sur les dimensions organisationnelles et institutionnelles. Ils concernent tant les syndicats et les entreprises que les politiques publiques et s'intéressent à certaines thématiques comme les stratégies des acteurs, le partenariat, la gouvernance des entreprises, les nouveaux statuts d'emploi, le vieillissement au travail, l'équité en emploi et la formation.

\section{Les activités du crises}

En plus de la conduite de nombreux projets de recherche, l'accueil de stagiaires postdoctoraux, la formation des étudiants, le CRISES organise toute une série de séminaires et de colloques qui permettent le partage et la diffusion de connaissances nouvelles. Les cahiers de recherche, les rapports annuels et la programmation des activités peuvent être consultés à partir de notre site Internet à l'adresse suivante: http:// www.crises.uqam.ca.

Denis Harrisson

Directeur

\section{Résumé}

Le potentiel d'innovation et de transformation de l'économie sociale : quelques éléments de problématique

À la question, « les entreprises d'économie sociale sont-elles plus porteuses d'innovations sociales que les autres? », une réponse positive est avancée assez spontanément, aussi bien par les promoteurs que par la plupart des chercheurs s'y intéressant. À partir de recherches que nous avons réalisées individuellement ou en équipe et en tenant compte des études que nous avons pu consulter dans le cadre de nos travaux sur l'économie sociale, nous tentons d'avancer quelques éléments de problématique pour répondre à nouveau à cette question. Dans un premier temps, nous revenons sur ce qu'il faut entendre par innovation sociale, étant donné que c'est le plus souvent en référence à ce type d'innovation que l'économie sociale est considérée. Dans un deuxième temps, nous examinons les approches théoriques, notamment les définitions de l'économie sociale, et dans un troisième temps les recherches empiriques.

Sous l'angle des approches théoriques, le potentiel d'innovation de l'économie sociale est évalué, sans grande nuance, comme très élevé ou au contraire comme très faible. Dans un cas, il s'agit des approches théoriques relevant, entre autres, de la sociologie, de l'économie politique ou encore des hétérodoxes en économie, qui définissent l'économie sociale comme contrainte à innover puisqu'elle tente de répondre à des besoins voire des aspirations non satisfaits par le marché ou l'État. Dans l'autre cas, il s'agit des approches néoclassiques qui ne retrouvent dans l'économie sociale, ni la propriété individuelle, ni le profit, qui représentent selon eux les principaux ressorts de l'innovation.

Selon les approches empiriques, les évaluations sont plus nuancées tenant compte, entre autres, des phases de développement. Si la création est généralement l'occasion d'innover, la diffusion puis le développement conduisent souvent, mais non inexorablement, à la banalisation, à l'isomorphisme institutionnel voire à la privatisation. Les études de cas qui ont porté surtout sur des secteurs relativement nouveaux, tels le travail, l'insertion et l'aide à domicile, laissent voir que le potentiel d'innovation n' est pas toujours actualisé, entre autres, faute de financements ou de soutien appropriés. Enfin, au moins une enquête quantitative tend à montrer que les associations dans le domaine de la santé et des services sociaux se distingueraient les innovations radicales que les innovations incrémentales que favorise la coopération entre parties prenantes.

Par ailleurs, lorsqu'on considère la dernière génération d'économie sociale comme un ensemble relativement continu d'initiatives de la société civile et en liaison avec les mouvements sociaux qui les ont inspirés, le potentiel de transformation semble l'emporter sur le potentiel d'innovation. Comme pour les générations précédentes, la " nouvelle » économie sociale a contribué grandement à « adoucir » les destructions engendrées par les innovations radicales, 
mais elle participerait plus qu'auparavant à la reconfiguration de l'État-providence et à l'émergence d'un modèle de développement où l'innovation deviendrait permanente et continue. Cette relecture d'un nombre significatif de travaux sur l'économie sociale, qui n'ont pas toujours été réalisés avec comme premier objectif l'étude des innovations, laisse supposer que l'approche en termes d'innovation et de transformation ne manque pas de pertinence pour mieux comprendre l'économie sociale mais aussi les grandes transformations en cours.

Benoît Lévesque

\section{Introduction}

La question du potentiel d'innovation de l'économie sociale semble difficilement dissociable de celle des transformations sociales (socio-économiques et sociopolitiques), surtout si l'on veut une évaluation relativement complète de ce potentiel. Sous cet angle, la comparaison avec les entreprises capitalistes ne saurait se faire terme à terme, sans aucune autre considération. Cela ne veut pas dire pour autant qu'il faille confondre innovation et transformation sociale puisque l'une renvoie à la fonction entrepreneuriale de l'économie sociale alors que l'autre désigne un type spécifique de changement à l'échelle sociétale. À la différence du changement social qui s'opère le plus souvent dans la continuité, à l'exemple de la croissance chez le vivant, les transformations sociales correspondent à des mutations mettant ainsi l'accent sur la discontinuité plutôt que la continuité. Dans la mesure où l'économie sociale représente plus qu'une somme d'entreprises en raison, entre autres, de la parenté des composantes ${ }^{1}$ et de leur rapport à des projets de société, sa capacité d'innovation peut être influencée par cette visée de transformation sociale. Cette dernière ouvre, aux entreprises et aux organisations de l'économie sociale, des pistes qu'interdirait la logique du seul calcul comptable, a fortiori à la rentabilité maximale. Enfin, l'économie sociale comme entité relativement spécifique au regard du secteur public et du secteur privé capitaliste peut contribuer à des transformations majeures, notamment suite à des périodes de grande crise telle celle amorcée à partir du milieu des années 1970 (EVERS ET LAVILLE, 2004; PERRI 6 et Vidal, 1994; DEFOURNY et MONZON, 1992).

À la question, « les entreprises d'économie sociale sont-elles plus porteuses d'innovations sociales que les autres ? », une réponse positive est avancée spontanément, aussi bien par les promoteurs que par la plupart des chercheurs s'y intéressant (LÉVESQUE, 2004; Osborne, 1994 et 1994a). Si plusieurs raisons peuvent justifier une telle réponse, il faut aussi admettre que certains chercheurs se montrent plus réservés, allant jusqu' à répondre négativement à la question. Dans ce court texte, nos objectifs sont modestes puisque nous proposons quelques éléments de problématique élaborés en grande partie à partir de revues de littérature réalisées séparément, soit dans un cas sur les innovations sociales (LÉVESQUE, 2005; LÉVESQUE, BOURQUE et FORGUES, 2001), dans l'autre sur l'économie sociale avec d'autres collègues (LÉVESQUE et MENDELL, 2004; LAVILLE, LÉVESQUE et MENDELL, 2005; LÉVESQUE, 2005; JETTÉ, LÉVESQUE, MAGER et VAILLANCOURT, 2000; LÉVESQUE et MALO,1995), sans négliger pour autant les recherches réalisées par d'autres chercheurs. Dans le meilleur des cas, ces éléments de problématique pourraient contribuer à la construction d'un cadre théorique concernant le potentiel d'innovation et de transformation de l'économie sociale. Dans cette visée, nous traiterons d'abord de l'innovation sociale comme telle (1), puis du potentiel d'innovation de l'économie sociale (2), à partir d'approches théoriques (2.1) et de recherches empiriques (2.2), pour conclure sur des considérations concernant le potentiel de transformation sociale.

\section{Les innovations sociales}

En dépit de quelques recherches réalisées antérieurement (COLEMAN, 1970; GABORD, 1970; HALL, 1977; TAYLOR, 1970; MOSS, 1982; WHYTE, 1982), les chercheurs n'ont analysé 
que fort récemment l'innovation sociale en référence explicite aux travaux fondateurs de Schumpeter (1939). L'économiste d'origine autrichienne a fourni plusieurs éléments intéressants, à commencer par une typologie des innovations, qui permet de distinguer les innovations de produit, de procédé, de marché et de nouvelles organisations ou combinaisons. Dans le domaine manufacturier, ces innovations font appel à la technologie et/ou au marché, à l'exception des nouvelles formes d'organisation (ex. celles touchant l'organisation du travail et la gestion de la qualité), qui sont définies comme innovations organisationnelles ${ }^{2}$ par le Manuel d'Oslo (3ième édition, 2005) et comme innovations sociales par les économistes hétérodoxes (FREEMAN, 1991). On comprend que, parmi les premières définitions de l'innovation sociale, on retrouve celle d'innovations organisationnelles que les économistes institutionnalistes et les sociologues compléteront avec les innovations institutionnelles pour couvrir le domaine des institutions, des normes, de la réglementation et plus largement des formes de régulation (THÉRÊT, 2000; CORIAT et WEINSTEIN, 1995; BÉLANGER, GRANT et LÉVESQUE, 1992; BÉLANGER et LÉVESQUE, 1992). Les recherches sur les entreprises ont ainsi montré que les innovations sociales pouvaient exister dans le domaine de la production et plus largement dans le domaine du développement économique (BÉLANGER, LAPOINTE et LÉVESQUE, 2004).

La distinction entre innovations radicales et innovations incrémentielles, qui est également basée sur l'œuvre de Schumpeter, peut être adaptée pour une meilleure compréhension des innovations sociales. Les innovations radicales sont en quelque sorte en rupture avec ce qui existe, transcendant les limites technologiques qui s'imposaient jusque-là (FREEMAN, 1982) alors que les secondes, les innovations incrémentielles, se veulent plutôt des innovations progressives, « une série de changements dans le cadre des paramètres connus ou de l'introduction dans un produit donné de caractéristiques techniques déjà utilisées dans des produits similaires »(FAGERBERG, 2003, p. 5). Même si les innovations incrémentielles sont moins spectaculaires, plusieurs considèrent que l'impact cumulatif de ces innovations peut être aussi grand voire plus, puisque « le plus gros des bénéfices économiques vient des innovations incrémentielles et des améliorations » (Ibid). En revanche, on ne peut faire l'économie d'innovation radicale pour penser le rapport des innovations aux transformations sociales. D'autres notions intermédiaires doivent aussi être examinées.

Dans cette visée, les analyses évolutionnistes et institutionnalistes de l'innovation ont repris l'hypothèse de Schumpeter selon laquelle les innovations se produisent généralement en grappes, notamment dans les périodes de sortie de crise. Ainsi, les innovations, qui se multiplient alors, ne se font pas dans toutes les directions, mais selon un paradigme sociotechnique en émergence, soit à partir d'une nouvelle vision des problèmes et des solutions possibles (notion qui peut être élargie à celle de nouveau paradigme sociétal pour rendre compte des innovations sociales) (LIPIETZ, 1989). Conformément au nouveau paradigme, les innovations donneront lieu à une trajectoire d'innovation ou un sentier d'innovation, qui fera en sorte que le chemin parcouru (path dependency) produira de plus en plus d'irréversibilité. On comprendra que « la transition d'un paradigme technologique à l'autre peut durer longtemps » (PETIT et SOETE, 2003, p. 92), a fortiori quand il s'agit d'un nouveau paradigme sociétal (TOURAINE, 2005). En effet, pour s'imposer largement, le nouveau paradigme doit s'appuyer non seulement sur de nouvelles représentations (la capacité d'imaginer combinée à la capacité d'oublier) mais aussi des expérimentations réussies, soit des innovations réalisées à l'échelle micro (LUNDVALL, 1992). De plus, même si les innovations suscitent des imitations en raison de leur réussite exemplaire, elles ne se diffuseront à l'échelle de la société qu'avec l'aide de nouveaux arrangements institutionnels (lois, réglementations, institutions, ententes internationales, répartition des pouvoirs selon diverses échelles, programmes et mesures diverses) qui permettront de vaincre les résistances au changement d'une partie des acteurs concernés et de soutenir les innovateurs (BÉLANGER, LAPOINTE et LÉVESQUE, 2004; THÉRET, 2000). Ce qui laisse entrevoir l'importance de l'environnement institutionnel et sociétal.

La littérature récente sur les innovations technologiques et scientifiques attirera 
l'attention sur l'importance de l'environnement et des réseaux. Même si les innovations sont généralement produites dans le cadre d'entreprises et d'organisations, la notion de système social d'innovation nous invite à considérer que le potentiel d'innovation peut être favorisé grandement par l'environnement que constituent les universités, le système financier, les services offerts, les réglementations, la culture, la cohésion sociale, etc. Selon que le type d'environnement considéré, le "système d'innovation » sera restreinte au « système sociotechnique d'innovation », notamment les rapports entre les entreprises, les universités, les centres de recherche, l'État et le marché (NELSON et WINTER, 1982) ou plutôt élargi au " système social d'innovation » qui comprend non seulement le système sociotechnique mais aussi l'ensemble des institutions pouvant influer sur la production de biens ou de services dans une société (LUNDVALL, 1992); (AMABLE, BARRÉ et BOYER, 1997). Par la suite, les chercheurs ont utilisé cette notion pour caractériser le potentiel d'innovation propre à certaines industries ou à certains territoires, tel est le cas de la notion de « système régional d'innovation » très utilisée dans le domaine des sciences régionales (BRACXYK, COOKE et HEIDENREICH, 1998). Ces notions permettent aussi de caractériser l'environnement relativement spécifique d'un territoire ou d'un secteur sur le plan des innovations. Pour questionner l'importance des innovations dans le domaine de l'économie sociale de même que pour l'évaluation de leur potentiel, la notion de système social d'innovation mérite d'être prise en considération. Ainsi, Hall et Soskice (2001) dans leur ouvrage sur la diversité des capitalismes ont montré que, dans les pays d'économie de marché coordonné favorisant la concertation et la coopération, les firmes misant sur les innovations incrémentielles avaient plus de chance de performer alors que, dans les pays d'économie libérale de marché encourageant la flexibilité tous azimuts, les firmes pouvaient plus facilement performer en misant sur les innovations radicales souvent à dominante technologique, sans se soucier des conséquences sociales pouvant en résulter. Même si les innovations radicales sont par définition plus lourdes de changement et donc de "destruction créatrice », les innovations incrémentielles par amélioration continue peuvent être souvent plus déterminantes sur la performance des entreprises, notamment dans certains domaines.

Des recherches relevant de la sociologie et de l'histoire de la science et de la technologie montreront que les innovations ne sont pas linéaires comme on le supposait, soit recherche scientifique, invention, innovation, diffusion et adaptation, mais résulteraient d'une série d'ajustements mobilisant une pluralité d'acteurs de sorte que le résultat final est rarement donné au départ, d'où une grande incertitude (CALLON, 1989; LATOUR, 1989). En somme, les innovations résulteraient de processus sociaux, soit d'interactions et d'échanges $\mathrm{d}$ 'informations entre personnes, scientifiques, entrepreneurs, financiers, hommes politiques, usagers, clients formant ainsi des réseaux sociotechniques. Dans cette visée, il devient problématique d'établir des frontières rigides entre innovation technologique et innovation sociale: la première relève d'un processus social alors que la seconde ne peut se matérialiser sans base technique, ne serait-ce que l'utilisation du langage dans des lieux relativement déterminés. Enfin, pour Callon et Latour, la notion de réseau sociotechnique rend mieux compte des interactions entre les acteurs et les objets que celle de système d'innovation utilisée par les institutionnalistes, notion qui leur apparaît trop déterministe et rigide. À notre avis, la notion de réseau sociotechnique est effectivement moins rigide que celle de système $\mathrm{d}^{\prime}$ innovation parce qu'elle traduit le mode de production des innovations ou les processus menant à l'innovation telle que diffusée largement, mais cela n'enlève pas l'intérêt de considérer également la dimension plus institutionnelle de la production des innovations au même titre où innovations organisationnelles et innovations institutionnelles se complètent parce que désignant des réalités différentes, mais articulées (BÉLANGER et LÉVESQUE, 1991). L'innovation pourrait donc être comprise en termes de processus inscrits dans des systèmes sociaux d'innovation. Outre le fait qu' elles mettent bien en lumière le caractère social et collectif de toute innovation, les notions de système et de processus peuvent être très utiles pour établir des passerelles entre les innovations et les transformations sociales (BOYER, 1991).

Même s'il n'existe pas de frontière impénétrable entre innovation technologique et innovation sociale, il nous semble utile de les distinguer et de tenir compte de la spécificité du 
développement social, notamment en ce qui concerne les services sociaux. Ainsi, les recherches sur les innovations dans les services sont arrivées à la conclusion qu'il existe une sorte de rupture avec l'industrie manufacturière (CST, 2003; Björkman (2004), au point où " l'innovation change de logique et de portée » (CALLON, LARÉDO et RABEHARISOA, 1997, p. 34). Outre son caractère intangible, le service, notamment dans le domaine des services aux personnes et des services sociaux, se caractérise par le fait que l'usager est coproducteur du service avec le professionnel chargé de la prestation (GADREY, 1992; AUCLAIR et LAMPRON, 1987). Dès lors, la distinction entre produit et procédé devient « dénuée de signification, puisque le produit consiste précisément en sa fabrication » (CALLON, LARÉDO et RABEHARISOA, 1997, p. 34). De plus, dans le cas des services, les innovations de produit deviennent souvent organisationnelles puisque « que la division des tâches, leur contenu, la nature des compétences, les procédures de coordination sont profondément remaniés et reconfigurés lorsque le contenu de la prestation est modifié » (Ibid, 35). Enfin, la distinction entre activité de conception et activité de production devient également peu significative « lorsqu'il s'agit de répondre à une demande encore incomplètement formulée et qui se construit dans l'interaction, la conception devient collective et elle se confond avec la réalisation de la prestation et les apprentissages croisés qu'elle suppose. » (Ibid). Tout cela n'est pas sans conséquence sur les rapports entre innovation et productivité, innovation et recherche, innovation et technologie. Par conséquent, de nouveaux services (ou produits) peuvent aussi être considérés comme des innovations sociales puisqu'ils consistent parfois en de nouvelles modalités d'intervention, de nouvelles façons de faire, de nouvelles formes organisationnelles, de nouvelles relations sociales (LAVILLE, 2005).

De plus, dans le cas des services non marchands, la validation directe ou indirecte par le marché, qui permet selon Schumpeter de distinguer l'invention de l'innovation, est remplacée par la redistribution assurée par les pouvoirs publics et complétée dans bien des cas par la réciprocité ou même une contribution monétaire de l'usager. On comprend ainsi l'importance de l'institutionnalisation pour assurer une validation des innovations sociales, notamment celle résultant de nouveaux services. Ces éléments comme les précédents se retrouvent dans la définition proposée par le Groupe de travail sur l'innovation et reprise par la suite par le Ministère de la science et de l'innovation dans son énoncé de "politique québécoise de la science et de l'innovation » (Gouvernement du Québec, 2001, p. 11). L'innovation sociale y est définie comme « toute nouvelle approche, pratique, ou intervention, ou encore tout nouveau produit mis au point pour améliorer une situation ou solutionner un problème social et ayant trouvé preneur au niveau des institutions, des organisations, des communautés »(BOUCHARD, 1997, p. 7). Cette définition reprend également les éléments provenant de Schumpeter pour catégoriser les innovations, tels ceux de nouvelle combinaison (« nouvelle approche, pratique ou intervention »), de nouveau produit ou de nouveau service. Elle élargit le domaine des innovations sociales en y incluant non seulement les innovations organisationnelles mais aussi les produits ou les services qui visent la solution de problème sociaux et qui sont généralement dans le domaine non marchand. Elle reconnaît la nécessité pour l'innovation de trouver preneur, mais indique explicitement que cette opération peut se réaliser par d'autres médiations que celle du marché, soit par celle des institutions, des organisations et des communautés, à travers la redistribution et la réciprocité. Sans nier la possibilité d'innovation sociale dans le domaine du développement économique, cette définition s'est surtout préoccupée de rendre compte des innovations sociales dans le développement social. Enfin, comme nous l'avons suggéré, il y aurait avantage pour une meilleure compréhension de l'innovation sociale de tenir compte (avec les adaptations nécessaires) de l'ensemble des concepts mis de l'avant, au cours des dernières décennies, pour l'analyse de l'innovation.

\section{Le potentiel d'innovation de l'économie sociale}

Les recherches sur les innovations sociales dans le domaine de l'économie sociale peuvent être regroupées en deux catégories : des recherches à dominante théorique, notamment celles 
concernant la définition de l'économie sociale, et des recherches empiriques constituées pour la plupart d'études de cas, d'entreprises, d'organisations ou de secteurs, parfois avec une grille d'analyse incluant explicitement les innovations sociales (COMEAU, 1996). Comme nous le verrons, les conclusions sont sans doute plus nuancées dans le cas des recherches empiriques que dans celui des recherches théoriques qui ont tendance à conclure positivement ou négativement, mais sans grande nuance. Il va de soi que les recherches empiriques sont plus difficiles à circonscrire alors que la production théorique est plus restreinte et souvent redondante.

\subsection{Point de vue théorique : l'économie sociale fortement ou faiblement innovante?}

La spécificité institutionnelle et organisationnelle de l'économie sociale a d'abord été définie de manière variable selon les approches, comme nous l'avons montré ailleurs (LÉVESQUE et NINACS, 1997; LÉVESQUE et MENDELL, 1999). La plupart des définitions apportées par les chercheurs laissent voir plus ou moins explicitement comment les entreprises d'économie sociale comme type-idéal peuvent être innovatrices. C'est le cas de la définition de l'économie sociale proposée par Vienney (1980, 1981 et 1994), définition qui sera la plus diffusée dans le monde francophone, au moins jusqu'à tout récemment. Elle est aussi la plus complète puisque l'économie sociale y est définie à partir de plusieurs éléments - catégorie $\mathrm{d}$ 'acteurs, activités économiques et ensemble de règles - formant un système dans le cadre $\mathrm{d}$ 'une formule combinant un groupement de personnes et une entreprise réunis dans un double rapport de sociétariat et d'activité. Il en résulte que l'entreprise d'économie sociale constitue au départ une matrice d'où l'innovation peut surgir dans au moins trois directions. En premier lieu, l'économie sociale innove en donnant, à certains acteurs relativement dominés, la possibilité d'accéder au pouvoir de l'entrepreneur, de réaliser des projets d'entreprises qu'ils n'auraient pu réaliser individuellement. En deuxième lieu, les activités mises en œuvre sont également nouvelles puisqu'il s'agit d'activités sans doute nécessaires mais délaissées par le marché ou l'État. En troisième lieu, les règles de fonctionnement sont également inédites dans le monde de la production de biens ou de services puisqu'elles visent à la fois le regroupement de personnes et l'entreprise (ou l'organisation) de même leur rapport sous l'angle Du sociétariat et de l'activité. Comme certains le lui ont reproché, Vienney considérait la coopérative comme le type idéal de l'économie sociale comme l'avait fait avant lui Charles Gide (DESROCHE, 1983).

Dans les années 1990, une nouvelle génération de chercheurs s'est efforcée d'élaborer une définition de l'économie sociale rendant compte plus explicitement des associations et des entreprises en émergence, notamment celles évoluant dans le développement social, les services de proximité et les services sociaux et de santé (LAVILLE, BORZEGA, DEFOURNY, EVERS, LEWIS, NYSSENS et PESTOFF, 2000 ; LAVILLE, 1994 ; EVERS,1995 ; PESTOFF, 1995 ; ENJOLRAS, 1994). En premier lieu, ces chercheurs mettent en lumière le rôle stratégique de l'impulsion réciprocitaire (et donc l'importance des ressources non marchandes et non monétaires), notamment pour l'émergence des services de proximité. En deuxième lieu, ils identifient une innovation pour le moins radicale, soit « la construction conjointe de l'offre et de la demande par les professionnels et les usagers », ce qui suppose la constitution de miniespaces publics laissant entrevoir une dimension politique souvent occultée en ce qui concerne les innovations sociales. En troisième lieu, ils s'appuient principalement sur Polanyi (1944) pour montrer que la nouvelle économie sociale repose sur une conception élargie de l'économie et du politique (DACHEUX et LAVILLE, 2004; LAVILLE, 1994). La mobilisation d' une grande diversité de ressources (marchandes, non-marchandes et non-monétaires) permet de parler d'hybridation et donc d'économie plurielle alors que la participation citoyenne fonde en quelque sorte l'idée d'une économie solidaire. Ces approches théoriques mettent bien en lumière la spécificité institutionnelle de ces initiatives de la société civile et comment ces dernières constituent une matrice favorable à l'innovation sociale. Enfin, comme les termes de «mixed economy of social welfare » ou de «pluralism welfare » le laissent supposer, ce type 
d'organisation occupe un espace intermédiaire entre l'individu et l'État tout en s'inscrivant dans une régulation concernant aussi bien le marché, la société civile et le domestique (EVERS et LAVILLE, 2004, p. 15). Sous cet angle, la " nouvelle économie sociale » participerait à la constitution d'un « nouveau régime de gouvernance de l'intérêt général » où seraient mobilisés de façon inédite l'État et ses agences, le marché à travers les entreprises et la société civile, à travers, entre autres, les associations volontaires (ENJOLRAS, 2004).

Ces diverses définitions qui centrent leur attention, dans un premier temps, plus sur les coopératives et, dans un deuxième temps, sur les associations et les entreprises sociales, laissent supposer que l'économie sociale est un « laboratoire d'innovations sociales », expression reprise dans de nombreux textes. Qu'il s'agisse de la définition de Vienney ou de celles plus récentes, le potentiel d'innovation serait maximal au moment de la création qui se fait selon une forme institutionnelle plus ou moins codifiée par la législation. D'un point de vue théorique, certains ajoutent d'autres arguments complémentaires telle la proximité des collectivités locales et des lieux d'appartenance que permet au groupement de personnes et par suite la possibilite d'ajustements relativement constants (innovation continue) ou encore la prise en charge conjointe du social et de l'économie, des externalités et du long terme qui permette de s'inscrire assez spontanément dans la logique du développement durable. Mais, ce potentiel d'innovation présent en principe lors de la création pourra s'atténuer en cours de la diffusion et de l'institutionnalisation comme le montreront les études empiriques. Enfin, la plupart de ces définitions ne suffisent pas à rendre compte des innovations dans les entreprises plus institutionnalisées (matures) d'économie sociale, notamment celles qui se trouvent en concurrence avec les entreprises capitalistes, telles les grandes coopératives agricoles (CÔTÉ, 2003) et les grandes banques coopératives (TREMBLAY et CÔTÉ, 2001).

Par ailleurs, il existe également des analyses théoriques, notamment celles d'économistes néo-classiques les plus orthodoxes, qui considèrent que les coopértives et des associations sans but lucratif sont en principe moins innovatrices pour au moins trois raisons (OSBORNE, 1994; LEPAGE, 1976 et 1978; PERRI 6, 1993, p. 397-8; ZIMMERMANN, 1999). En premier lieu, les entreprises sans propriétaire individuel ou à propriété collective, comme c'est le cas des coopératives et des associations, ne sauraient être innovatrices en raison d'une structure de propriété qui n'encourage par l'innovation et la prise de risque (JENSEN et MECKLING, 1979). En deuxième lieu, ces entreprises manquent d'incitatifs appropriés pour innover et prendre des risques, notamment la recherche maximale du profit qu'on retrouve d'autant moins que l'économie sociale vise un arbitrage entre l'économique et le social. En troisième lieu, l'économie sociale émerge dans des secteurs délaissés par le marché ou par l'État de sorte que la plupart d'entre elles évoluent dans des secteurs non ou faiblement rentables. Enfin, la plupart des entreprises d'économie sociale se retrouvent dans des secteurs faiblement concurrentiels ou encore dans des niches ou des marchés protégés, ce qui ne semble pas être le cas que des coopératives plus anciennes, notamment dans le secteur de l'agriculture et des finances.

Sans essayer de réfuter tous ces arguments théoriques, on peut faire appel à Schumpeter (1939) ou encore à Weber (1964) pour montrer que la motivation profonde de l'entrepreneur capitaliste, celui qui par définition innove, relève rarement du seul calcul rationnel. Selon ces deux auteurs classiques, la logique de l'entrepreneur serait constituée d'un mélange d'éléments rationnels et émotionnels: d'une part, des éléments rationnels telles la prévision et l'inscription dans un environnement rationnel où l'on retrouve l'argent, la science, le droit; d'autre part, des éléments irrationnels telles l'émotion et la passion que suscite le rêve de fonder une dynastie, une grande famille, un empire (LÉVESQUE, 2002). C'est pourquoi l'entrepreneur, dans la mesure où il s'est fait innovateur, a bien souvent été considéré comme un déviant par rapport aux normes dominantes (SCHUMPETER, 1939).

De plus, une meilleure compréhension de l'entreprise et des organisations a amené certains analystes à proposer une vision de l'entrepreneur centrée moins sur sa personnalité que sur les mécanismes qui rendent son projet possible. En définissant l'action d'entreprendre comme celle de mobiliser des moyens et des personnes « afin de constituer une structure de 
production, ou de faire évoluer une structure, par innovation », Gomez conclut que l'action d'entreprendre consiste principalement «à convaincre les acteurs nécessaires à la réalisation d'un objet commun » (GOMEZ, 1996, p. 224). Dès lors, « si l'entrepreneur réussit, c'est qu'il a contribué à construire (ou modifier) un système d'information sur l'objectif commun d'efficience, qui s'est avéré plus cohérent que celui de tout système alternatif » (Ibidem, p. 228). Par suite, l'entrepreneur peut être défini comme « un artisan du système de conviction » ou encore comme quelqu'un qui réussit à créer du sens à partir d'un projet. Dans cette visée, on peut supposer que l'entreprise ou l'organisation de l'économie sociale (les coopératives et les OBNL produisant des biens et des services) n'est pas en déficit de signification par rapport à l'entreprise capitaliste puisqu'elle fait appel à des valeurs et des convictions. En somme, d'un point de vue théorique, il est possible de soutenir que l'entrepreneur social ou même collectif ne manque pas d'incitatifs pour innover, même si ces incitatifs ne sont pas financiers (BORZAGA et DEFOURNY, 2001; LAVILLE et NYSSEN, 1998).

\subsection{Point de vue empirique : un potentiel qui n'est pas pleinement actualisé}

Au moment même où la notion d'économie sociale a été redécouverte en France et en Belgique, les promoteurs n'hésitent alors pas à mettre de l'avant sa capacité d'innovation pour justifier sa pertinence, comme le suggère la création en 1976 du Centre d'information sur les innovations sociales (JEANTET et VERDIER, 1982, p. 40) de même que la proposition d'un Centre de recherches et d'innovations européen de l'économie sociale qui ne verra pas le jour (CNLAMCA et CIRIEC, 1979, p. 79-80). Cette conviction des promoteurs d'associations (non profit organization) est également forte dans le monde anglo-saxon, notamment depuis les années 1970 (OSBORNE, 1994). Dans les recherches empiriques et plus académiques sur l'économie sociale, la référence aux innovations sociales est assez constante mais les conclusions nous semblent beaucoup plus nuancées.

Pour plusieurs raisons, il faut avouer qu'il est difficile de dégager de ces recherches des conclusions bien fermes quant à la concrétisation du potentiel d'innovation de l'économie sociale. En premier lieu, très peu de ces recherches se sont efforcées de définir au plan conceptuel l'innovation, a fortiori l'innovation sociale. En deuxième lieu, les recherches les plus nombreuses sont à dominante qualitative, soit des études de cas (entreprises, associations, secteurs), auxquelles s'ajoutent des recherches sur des thèmes ou des questions reliées à l'émergence de la «nouvelle économie sociale ». Ainsi, une analyse de la documentation de langue française comprenant 811 références bibliographiques permettait de conclure que « la question des acteurs sociaux et des processus institutionnels inhérents à l'émergence de la nouvelle économie sociale ainsi qu'au partage des responsabilités dans le domaine de la santé et du bien-être ont été des thèmes de prédilection » pour la période 1990-2000 (JETTÉ, LEVESQUE, MAGER et VAILLANCOURT, 2000, p. 72). À moins de disposer de moyens importants, il faut admettre qu'il n' est pas possible de rendre compte de manière exhaustive de la production internationale dans ce domaine. Tout au plus, nous pouvons illustrer la pertinence d'une approche en termes d'innovation sociale, à partir de recherches auxquels nous avons été associé de près ou même de loin. Enfin, en plus des différentes composantes de l'économie sociale, il faut également tenir compte de la diversité des rapports que ces entreprises entretiennent avec le marché (entreprises devant relever le défi de la mondialisation, entreprises évoluant dans des niches, etc.) ou encore avec l'État (régulation concurrentielle, régulation tutélaire, régulation partenariale).

Ainsi, les entreprises coopératives évoluant dans des domaines ouverts à la concurrence mondiale comme l'agro-alimentaire et les finances se doivent d'être aussi innovatrices que les entreprises capitalistes sous peine de disparaître. C'est d'ailleurs ce qui est arrivé à de nombreuses coopératives de consommation qui sont disparues et à également de nombreuses mutuelles qui se sont privatisées. Il faut donc tenir compte des orientations stratégiques prises 
par les dirigeants et des phases de développement des entreprises pour évaluer la portée des innovations mises de l'avant. Comme l'ont montré Malo et Vézina, ensemble ou séparément (MALO et VÉZINA, 2004; MALO, 2001; VÉZINA, 2003), les entreprises coopératives, comme d'ailleurs bon nombre d'entreprises et d'organisations d'économie sociale, traversent au moins trois phases de développement. Sous l'angle des stratégies de création de valeur et de configurations organisationnelles, les auteures identifient trois phases: une première phase, celle de la création de l'entreprise, qui constitue une innovation en soi; une seconde phase qui est celle de la diffusion de l'innovation; une troisième phase plus complexe où l'évolution peut se faire selon l'une ou l'autre des trois directions suivantes: celle de la focalisation par spécialisation dans des services peu standardisés pour un segment de ses clients-usagers, ce qui représente un retour à l'innovation; celle de la standardisation qui résulte d'une stratégie d'imitation des entreprises capitalistes et donc peu innovante; celle enfin de l'hybridation où les dirigeants recherchent une sorte d'équilibre entre focalisation et standardisation. Les choix à l'origine de chacune de ces phases représentent des défis importants sous l'angle de l'innovation et de la cohésion association-entreprise. Ainsi, la standardisation peut conduire à la banalisation à travers, entre autres, un isomorphisme institutionnel.

Ce cadre théorique concernant les phases de développement des entreprises ou des organisations d'économie sociale permet de dégager un espace pour des innovations stratégiques et structurelles indispensables à la fois pour la réussite des affaires (la création de valeur économique) et pour le maintien de la spécificité coopérative (la création de valeur coopérative) (VÉZINA, 2003a). Pour les entreprises matures qui désirent miser sur les innovations sociales, le défi est alors de conjuguer positionnement (marché) et perspective (éthique et utopie) : " sans perspective, sans projet de société, sans visée vers le meilleur des mondes, beaucoup de stratégies de positionnement par la différenciation sont en fait des stratégies d'imitation des joueurs dominants » (MALO, 2001, p. 92). Mais, ne retenir que la perspective sans tenir compte des règles du secteur peut conduire sans doute à la banalisation, mais aussi à la privatisation et, dans certains cas, à la faillite comme ce fut le cas des Pêcheurs-Unis au Québec (LAROCQUE, 1988). En somme, "seule une articulation du volontarisme et du déterminisme, de la perspective de transformation sociale et du positionnement économique rend le processus stratégique porteur à la fois d'identité et de viabilité » (MALO, 2001, p. 93). Enfin, comme on peut l'entrevoir, les choix stratégiques peuvent donner lieu non seulement à des innovations organisationnelles mais aussi à des innovations institutionnelles (CÔTÉ, 2001). En effet, pour le meilleur comme le pire, les grandes entreprises coopératives et mutuellistes ont souvent la capacité d'influer sur les pouvoirs publics pour modifier leur cadre institutionnel afin qu'il soit en cohérence avec leurs orientations stratégiques (ZEVI et MONZON, 1995; LÉVESQUE et CÔTÉ, 1995; LÉVESQUE et MALO, 1995 et 1997).

Dans cette visée, le cas du Mouvement Desjardins au Québec est intéressant puisqu'il a dû procéder à une réingénierie des processus d'affaires de ses caisses, comme toutes les banques l'avaient fait avant lui, de même qu'à une rationalisation de ses activités et une réorganisation de sa structure organisationnelle et institutionnelle (LÉVESQUE et MALO, 1995 et 1997). En dépit des critiques relativement vives formulées à l'époque, Desjardins s'est distingué des banques moins par ses innovations technologiques que par ses innovations sociales, sa façon de réaliser sa modernisation. En effet, les dirigeants ont accepté au départ de prendre beaucoup plus de temps pour réaliser cette opération qu'ils ont planifié sur une période de cinq ans (et qui s'est déroulée même au-delà), de s'engager à protéger les emplois (sans licenciement) et à procéder par étapes, à commencer par une expérimentation dans un nombre restreint de caisses, les caisses vitrines, et en faisant des investissements de près d'un demi milliard de dollars pour la formation et la reconversion d'une partie du personnel (LÉVESQUE, BÉLANGER et MAGER, 1999 et 1997), sans oublier une opération de Renouveau coopératif. Enfin, la mise en place d'une fédération unique entraînant la disparition des fédérations régionales (et de la fédération des caisses d'économie) fut également un processus qui s'est fait en misant sur la participation et le débat, même si cela ne s'est pas fait sans 
tensions et parfois conflits. De même, la réduction du nombre des caisses locales, qui sont passées de 1200 à environ 600 en quelques années, a sans doute suscité des débats et des tensions, mais la formule de centres de services et la création de centres financiers aux entreprises (CFE) ont permis de réaliser une reconfiguration majeure de l'offre de service et, dans bien des cas, une amélioration de la qualité. À n'en pas douter, cette modernisation présente des similitudes avec celle réalisée dans les banques mais la grande différence nous semble provenir de la façon de le faire, notamment en mettant à contribution les membres à travers les instances de la démocratie représentative (en somme, en faisant appel à des innovations sociales). Les nombreuses recherches sur le Mouvement Desjardins et les caisses mettent également en lumière des innovations sociales passablement diversifiées, sans oublier des initiatives de partenariat avec des organisations relevant de la «nouvelle économie sociale » et le soutien du Chantier de l'économie sociale (MALO et alii, 2001; IGNATIEFF et MALO, 1997; MALO, 1997; LÉVESQUE et MENDELL, 2004).

Au cours des deux décennies, le secteur financier a été le lieu de nombreuses créations d'entreprises d'économie sociale (LÉVESQUE et MENDELL, 1998), dont certaines sont devenues assez rapidement des entreprises matures. Ainsi, le Fonds de solidarité des travailleurs (FTQ) créé en 1983 a constitué au départ une véritable matrice d'innovations entraînant un renouvellement à la fois du syndicalisme et des pratiques de financement des entreprises (LÉVESQUE, BÉLANGER, BOUCHARD, MENDELL et alii, 2000). D'abord l'idée qu'une grande centrale syndicale mette sur pied un fonds de capital de risque pour investir dans des PME, dans bien des cas non syndiquées, à partir de l'épargne salariale relevait de l'impensable. Ainsi, les premiers travailleurs, qui y contribuèrent, le firent pour la plupart par solidarité avec leur président alors que d'autres n'hésitèrent pas à dénoncer une telle initiative. Cette innovation radicale pour le monde syndical le fut également pour le monde financier. D'abord, la transformation de militants syndicaux en courtiers ouvriers pour recueillir l'épargne au sein même des entreprises de même que la formation économique des travailleurs, dans les entreprises où le Fonds avait investi, contribuèrent effectivement à transformer les pratiques syndicales. Ensuite, l'idée de n'investir dans les entreprises qu'à la suite d'un bilan social bilan réalisé par des employés proches de la centrale syndicale (voire même y provenant) et jugé indispensable pour que la proposition d'affaires soit retenue - devait apparaître dans le monde du capital de risque non seulement comme nouvelle mais également de nature à réduire le risque financier, notamment les aléas moraux. Enfin, la gouvernance du Fonds de solidarité, qui met en présence à la fois deux mondes, celui des dirigeants de grandes fédérations syndicales (les unions) et celui des financiers, a représenté de l'avis de tous une tension structurelle qui s'est révélée après coup un ingrédient déterminant de la matrice d'innovation. L'expertise syndicale dans la négociation des conflits expliquerait que cette tension structurelle ait pu être génératrice d'innovations plutôt que de luttes sans fin.

À partir des années 1990 le Fonds de solidarité des travailleurs s'est en quelque sorte imposé au Québec comme un acteur financier incontournable tout en demeurant généraliste, ce qui ne l'a pas empêché de créer plus d'une centaine de filiales avec leur propre conseil d'administration pour répondre aux besoins des régions et des collectivités locales, sans négliger pour autant des secteurs plus spécialisés relevant aussi bien de l'économie traditionnelle, tel le récréotouristique, que la nouvelle économie, telle la biotechnologie. Au lieu de répondre simplement aux demandes, il cherchera à partir de la seconde moitié des années 1990 à produire des effets structurants sur certains secteurs prometteurs en termes d'emploi, un peu comme s'il avait tenté de suppléer à l'absence de politiques industrielles ou à les influencer dans le sens de la création d'emploi. Au lieu d'imiter les autres institutions financières, ces dernières ont tenté plus souvent le contraire, soit emprunter les nouveaux sentiers qu'il avait ouverts. Après quelques années difficiles, il connaît à nouveau des rendements positifs, mais le maintien voire le renouvellement des nombreuses innovations semble constituer maintenant son principal défi, d'autant plus que l'environnement s'est modifié considérablement depuis quelques années. Ce cas montre toutefois comment une création, dans un domaine comme le financement des entreprises, peut atteindre en moins 
de vingt ans la stature d'une entreprise mature et fortement institutionnalisée. Enfin, à l'échelle du Canada, plus d'une vingtaine de fonds de travailleurs ont été mis sur pied entre 1990 et 1998. Au Québec, le Fondaction pour la coopération et l'emploi (CSN) créé en 1996 innove à la fois par son insertion dans les outils collectifs de la CSN et par son orientation en faveur de la coopération et du développement durable.

$\mathrm{Au}$ « milieu des années 1970 et surtout dans les années 1980, on a assisté à la multiplication de nouvelles coopératives qui, dans un contexte de crise économique, s'attachaient à préserver ou à créer de l'emploi dans le respect d'une certaine démocratie économique » (DEFOURNY, 2001, p. 51).

Ce renouveau coopératif dans le domaine du travail puis des services contribuera au moins en France et en Belgique «à donner une nouvelle jeunesse au concept déjà ancien d'économie sociale »(DEFOURNY, 2001, p. 51I). Au Québec, les coopératives de travail représentent exclusivement un secteur émergent puisque la quasi totalité d'entre elles ont été créées à partir de 1975, à l'exception des forestières et des coopératives de taxi (LÉVESQUE, CÔTÉ, CHOUINARD et RUSSELL,1985, p. 27). La coopération du travail y regroupait également des entreprises dites alternatives (JOYAL, 1989) comme en témoigne le fait que l'ancêtre de la Fédération des coopératives de travail créé en 1986 s'appelait Comité provincial de coopératives production, de travail et de pré-coopératives. Ainsi, Tricofil, une entreprise autogérée créée en 1976 et bien identifiée à la coopération du travail, n'était pas une coopérative $^{3}$. Avec l'adoption de modifications ${ }^{4}$ à la loi des coopératives en 1984, la mise en place d'un régime d'investissement coopératif (RIC) et la création de groupes conseils (Coopératives de développement régional), le développement des coopératives de travail sera facilité (LÉVESQUE, CÔTÉ, CHOUINARD et RUSSELL, 1985, p. 28). S'il existe peu de recherche sur ces coopératives en termes d'innovation, le terme innovation est par ailleurs presque toujours mentionné, à un moment ou l'autre.

Pour plusieurs analystes, la coopération du travail est la plus complète et la plus exigeante puisqu'elle suppose un engagement continu et intensif des membres à la différence de la plupart des coopératives de consommation (FERRERIA, 2004). Il est par ailleurs plus difficile $\mathrm{d}^{\prime}$ en assurer la diffusion puisqu'elles naissent dans une grande diversité de secteurs de sorte qu'il n'est pas facile de créer des regroupements sectoriels susceptibles de redynamiser l'activité économique des entreprises situées à la base. Ainsi, même si le Québec est considéré comme un chef de fil au Canada dans le domaine des coopératives de travail (CRADDOCK et KENNEDY, 2005), la diffusion de ce type de coopératives y demeure modeste ${ }^{5}$ de même que celle des coopératives de travailleurs actionnaires, une innovation québécoise s'approchant de la formule américaine des ESOP, sur lesquelles les prometteurs comptaient faire une percée ${ }^{6}$. Toute proportion gardée, le Royaume-Uni avec un peu plus de 1000 et la France avec environ 1550 coopératives de travail ne font pas meilleure figure que le Québec alors que l'Italie et surtout l'Espagne avec 14000 coopératives de travail révèlent que, moyennant certaines conditions, la formule peut connaître du succès (CRADDOCK et KENNEDY, 2005; CIRIEC Espana, 2002, p. 36).

Par ailleurs, les recherches empiriques sur les coopératives de travail laissent voir que leur potentiel d'innovation est très inégalement actualisé. Dans une recherche à l'échelle de l'Europe, Laville (1993) distingue deux types de coopératives de travail, celui reposant sur des collectifs volontaires (groupes fusionnels), qui ont émergé surtout à partir de la fin des années 1960, et celui donnant lieu à des collectifs contraints (groupes clivés), qui ont été formées principalement à partir des années 1980. Dans le premier cas, la formation du collectif "se caractérise par l'enthousiasme coopératif », un fort engagement des membres, y compris financier, une stratégie de formation professionnelle, la démocratie directe, l'absence de hiérarchie et l'égalitarisme qui peut parfois compenser la fragilité économique. En effet, « l'effet de créativité sur le produit, stimulé par la nature participative des structures d'organisation choisies, rend ces collectifs d'autant plus efficaces qu'ils exercent des activités où les produits sont à haute technicité » (LAVILLE, 1993, p. 10). On passerait ainsi de " l'utopie alternative à l'entreprise innovante ». Dans le deuxième cas, celui des collectifs 
contraints, où les membres se mobilisent d'abord pour sauver ou maintenir leur emploi, représente un ensemble plus hétérogène bien que concentré dans des secteurs plutôt traditionnels. Dans ce cas, les succès semblent reposer moins sur l'innovation que sur la réduction des coûts par « le recours à la flexibilisation qualitative » et par une gestion réussissant à concilier " projets économiques et sociaux, urgences à court terme et visées à long terme » (Ibid, p. 20). Enfin, toujours dans ce deuxième cas, la démocratie tend à se limiter à la démocratie représentative voire à une démocratie purement formelle.

Une recherche réalisée sur un échantillon plus restreint de coopératives québécoises met également en relief l'hétérogénéité des coopératives de travail au plan de l'innovation. Ainsi, Comeau (1993) identifie trois types de coopératives de travail: des " coopératives idéologiques » qui valorisent les principes coopératives avant le profit; des coopératives participantes qui maintiennent « les principes démocratiques jusqu'à ce que les conditions économiques se détériorent "; des PME coopératives qui n'encouragent pas la participation et l'engagement des travailleurs, faiblement innovante (COMEAU, 1993, p. 36). Cette recherche et d'autres montrent bien qu'une forme institutionnelle relativement innovante, qui fait des travailleurs les propriétaires collectifs de l'entreprise, $n^{\prime}$ entraîne pas nécessairement des innovations organisationnelles, notamment au niveau de l'organisation du travail et de la gouvernance de l'entreprise (COMEAU et LÉVESQUE, 1993). Sur le plan de l'organisation du travail et donc des innovations organisationnelles, plusieurs entreprises capitalistes auraient été plus performantes que certaines coopératives de travail, en dépit de la supériorité institutionnelle de ces dernières. Autrement dit, « la conviction d'avoir un processus de décision démocratique » peut empêcher de questionner et par suite s'accompagner "d'inégalités engendrées par l'organisation du travail et de la production » (LAVILLE, 1998, p. 333)

Pour la "nouvelle économie sociale ", le domaine de l'insertion serait l'un des plus innovateurs des trente dernières années (DEMOUSTIER, 2000, p. 40) et l'un des plus représentatifs de l'économie sociale, au point où, certaines régions, telle la Flandre (en Belgique), considèrent l'économie sociale comme synonyme d'insertion (DEFOURNY, NYSSENS, SIMON, 1998). Même si les organisations et les entreprises s'y rattachant ne sont pas les plus nombreuses, ni les moins problématiques, elles constitueraient une sorte de " laboratoire d'innovations sociales », une matrice d'innovations mettant en relations plusieurs éléments qui ne le sont généralement pas, ce qui aurait pour effet de produire de l'inédit. À l'origine, l'insertion concernait des publics cibles, telles les personnes handicapées physiques et mentales, mais avec la crise économique elle «va peu à peu concerner toutes les catégories de population rendues vulnérables à la fois au niveau du travail et au niveau des liens sociaux, par le chômage et ses effets en termes d'exclusion sociale " (FECHER et alii, 2002, p. 17). Aux entreprises alternatives et aux coopératives de travail, qui tentaient de dépasser le salariat, s'ajouteront « des initiatives associatives modelées sur l'entreprise coopérative sans toutefois en adopter le statut » (FAVREAU, p. 167), qui passeront à « l'insertion dans le salariat » par l'amélioration des compétences et de la formation, par la formation et le soutien « aux chômeurs à la gestion à la gestion d'activités occupationnelles puis directement productives » (DEFOURNY, FAVREAU et LAVILLE, 1998, p. 48). Dans le cadre d'une entreprise soumise aux contraintes du marché, les entreprises d' insertion chercheront donc à « procurer pour une durée limitée des opportunités de travail et de formation » ou encore à offrir " une mise en situation de travail formatrice et socialisatrice » (Ibid, p. 340). Pour ces entreprises, qui doivent relever le défi du marché, une des difficultés sera d'assumer le surcoût que représente l'embauche de manière prioritaire de personnes exclues du marché du travail, ce qui entraîne une improductivité chronique, une rotation des travailleurs en insertion, sans oublier les coûts de formation et d'entraînement au travail. Dès lors, lorsque ces entreprises ont des activités à dominante marchande, elles devront faire appel à des ressources non marchandes, se mettre en relation avec les pouvoirs publics dont les programmes pertinents relèvent aussi bien des politiques d'emplois et de formation que des politiques sociales.

La recherche réalisée auprès de neuf pays par un groupe du CIRIEC international coordonné par Defourny, Favreau et Laville (1998) révèle l'importance, la diversité et 
l'hétérogénéité d'initiatives à dominante associative (mais non exclusivement puisqu'on y retrouve notamment des coopératives sociales) qui se sont développées surtout à partir du début des années 1980. Cette recherche, qui a mobilisé dix-sept chercheurs, permet d'identifier les principales innovations sociales de ce type d'entreprises et d'organisations. En premier lieu, ces initiatives associatives, qui ne recherchent pas le profit comme finalité, auraient permis une affectation plus efficace des ressources humaines en réduisant les coûts de sélection et de formation des personnes tout en révélant aux entreprises, futurs employeurs, les capacités productives de ce segment délicat du marché du travail, contribuant ainsi à améliorer le fonctionnement de ce dernier (BORZAGA, GUI, POVINELLI, 1998, p. 265). En deuxième lieu, l'économie sociale d'insertion innove dans son mode $\mathrm{d}^{\prime}$ " intervention » auprès des personnes exclues en misant sur leur participation active et en les reconnaissant comme membres à part entière d'une communauté de citoyens. De plus, dans le meilleur des cas, le choix de la participation active permet de déboucher sur la « co-production des actions entre professionnels et usagers », ce qui est à la base de l'auto-insertion (FECHER et alii, 2002, p. 290 ; EME, 1998; EME et LAVILLE, 1988). En troisième lieu, ces initiatives innovent non seulement en remettant en question le cloisonnement des politiques sociales et des politiques de l'emploi et de la formation, mais en créant un " espace inédit de traitement social du chômage » qui tente une articulation de l'ensemble de ces politiques avec une participation à la sphère économique. Ce faisant, elles révèlent que l'exclusion n'est pas exclusivement un problème social puisque les solutions doivent venir aussi bien de l'économique que du politique. Dans le cadre d'un État social actif, les pouvoirs publics, y compris à l'échelle de l'Union européenne, comprendront sans doute inégalement qu'il dispose « d'un véritable instrument novateur dans le domaine des politiques actives du travail » (EME, 1998, p. 287). En quatrième lieu, l'insertion par l'économie sociale, qui ne constitue pas comme tel un secteur d'activité et qui ne fait pas appel à un seul statut juridique, innove en donnant lieu à une pluralité d'activités, constituant ainsi une sorte de "laboratoire d'émergence de nouvelles activités » (DEMOUSTIER, 1998, p. 68). Ainsi, les premières coopératives sociales en Italie étaient des entreprises d'insertion qui ne servaient " pas les intérêts des seuls membres mais l'intérêt général de la collectivité à travers la promotion humaine et l'insertion sociale des citoyens » (BORZAGA, 1998, p. 104). En cinquième lieu, les initiatives d'insertion en économie sociale innovent également en faisant le lien avec le territoire de sorte qu'elles identifieront une forme d'exclusion géographique, des " territoires orphelins ", dans les zones rurales comme dans les zones urbaines (FONTAN, KLEIN et LÉVESQUE, 2003), ce qui donnera lieu non seulement à des entreprises territorialisées, mais aussi à des gouvernances locales et des outils de développement local ouverts sur le partenariat, comme on a pu l'observer au Québec, notamment avec les CDÉC, les SADC et les CLD (FAVREAU, 1998; LÉVESQUE, MENDELL, M'ZALI, MARTEL et DESROCHERS, 2003; COMEAU, FAVREAU, LÉVESQUE et MENDELL, 2001). Enfin, les initiatives d'insertion, comme d'ailleurs celles dans les services sociaux et de santé, innovent au plan institutionnel en donnant naissance à des entreprises sociales et à des hybridations des formes institutionnelles coopératives et associatives dans plusieurs pays (Belgique, Italie, France, Espagne, Portugal et Québec), soit des coopératives sociales qui s'ouvrent aux services à autrui et des entreprises sociales souvent constituées d'associations qui fonctionnement comme des coopératives mais sans le statut (BORZAGA et SPEAR, 2004; MUNKNER, 2004, GIRARD, 2004; MAC PHERSO, 2004; SPEAR, 2004).

Si les entreprises d'insertion dans le domaine de l'économie sociale représentent une sorte de « laboratoire d'innovations sociales » de par leur création, leur institutionnalisation relative forcément ce potentiel, d'autant plus que, même dans la période d'expérimentation, plusieurs limites ont été mises à jour, à commencer par une instrumentalisation par les pouvoirs publics ou encore une intégration à l'économie de marché au détriment de leur mission principale. De même, la multiplication de ces entreprises, qui se voulaient comme des cas favorisant l'entrée sur le véritable marché du travail, aurait eu comme conséquence de créer une nasse qui enferme ces «travailleurs » dans un second marché du travail pour précaires (DEMOUSTIER,1998). De plus, lorsque l'entreprise d'insertion offre des services de proximité, 
il existe une tension entre les travailleurs en situation d'insertion et les usagers ou clients qui veulent des services de qualité et une relation plus continue comme cela est manifeste dans le cas des services sociaux. Plus subtilement, les objectifs d'intégration que portent ces initiatives peuvent souvent masquer de nouvelles formes de domination dont les exclus sont l'objet de même que des dynamiques de fragmentation à l'oeuvre (EME, 1998). Enfin, il faut aussi reconnaître que plusieurs de sés entreprises sont d'une grande fragilité, en dépit de leur grand potentiel.

Enfin, « le secteur sanitaire et social est le secteur où l'Économie sociale est la mieux représentée » (DESMOUSTIER, 2000, p. 38), celui qui dans la plupart des pays regroupe le plus d'associations et de coopératives sociales (ENJOLRAS, 1995). Les recherches empiriques montrent bien le potentiel d'innovation de ces services d'économie sociale, potentiel souvent faiblement actualisé, mais accordent généralement plus d'attention à leur potentiel de transformation de l'État-providence qui leur semble plus facile à circonscrire. Â cette fin, les recherches adoptent souvent une perspective historique et comparative (EVERS et LAVILLE, 2004). Ainsi, dans la plupart des pays, les associations ont fait preuve d'innovation en défrichant les demandes sociales porteuses d'enjeux collectifs avant même que l'État social ne prenne forme. Par la suite, les associations seront relayées par l'État selon des modalités diverses. Si l'État-providence de type universaliste comme dans les pays scandinaves a eu tendance à laisser peu de place aux associations, l'État-providence corporatiste comme en Allemagne et en France les subventionne pour la prestation de services dans le cadre d'une régulation tutélaire qui réduit leur autonomie alors que l'État-providence dual comme l'Espagne et le Portugal se préoccupe moins des services publics, ne finançant les associations que sur des bases clientélistes, laissant souvent les services de soin aux familles (ENJOLRAS, 1995, p. 239; LAVILLE et NYSSENS, 2001, p. 235). Ces arrangements institutionnels seront remis en cause par la crise de l'État-providence, qui a suscité de nombreuses analyses, et le fait que, pour la prestation de services, les structures bureaucratiques et centralisées sont moins appropriées pour répondre à des besoins passablement différenciés comme c'est le cas des services sociaux et à fortiori des services d'aide à domicile. De plus, des facteurs sociaux tels le vieillissement de la population, l'entrée massive des femmes dans le salariat et la quasi généralisation de deux apporteurs de revenus par ménage contribueront à la multiplication d'initiatives de la société civile, soit d'associations pour répondre aux nouvelles demandes sociales dont celles des services à domicile.

La nouvelle vague des associations et des coopératives dans le domaine des services sociaux, à commencer par les services et l'aide à domicile, apporterait des innovations sociales d'un autre type que celles qui ont précédé l'État-providence (EVERS et LAVILLE, 2004). En premier lieu, les nouvelles associations répondraient non seulement aux échecs de l'État devant des demandes hétérogènes et aux échecs du marché devant l'asymétrie d'information dont les usagers sont l'objet avec l'offre marchande, mais aussi aux échecs des associations fondées sur la philanthropie et la charité dont les limites (insuffisance des ressources volontaires, particularisme, paternalisme et amateurisme) avaient justifié en partie la prise en charge par l'État (ENJOLRAS, 1995, p. 237). En deuxième lieu, les nouvelles associations sans but lucratif et les coopératives sociales, notamment à partir des années 1980, seraient le plus souvent des initiatives de la société civile ou l'on retrouverait une multiplicité de parties prenantes (VAILLANCOURT, AUBRY, JETTÉ, 2003, p. 161 et 296), soit, entre autres, des usagersclients, des travailleurs, des membres de la communauté et des acteurs du secteur public, d'où d'ailleurs de nouvelles formes institutionnelles telle la coopérative sociale et les entreprises sociales formées d'associations (SPEAR et BORZAGA, 2004; MUNKER, 2004; GIRARD, 2004; MACPHERSON, 2004). En troisième lieu, en introduisant une hétérogénéité des parties prenantes, ces nouvelles formes institutionnelles supposent également des innovations sociales pour dégager un intérêt général qui ne saurait résulter de la simple addition d'intérêts collectifs voire corporatistes. À partir de mini-espaces publics donnant la parole aux diverses parties prenantes pour un empowerment individuel et collectif, il devient possible de coconstruire une offre et une demande des services (innovation institutionnelle), qui constitue un préalable 
pour une coproduction nécessaire pour une prestation de services sociaux dont l'efficacité suppose des usagers actifs (innovation organisationnelle). En quatrième lieu, ces associations et coopératives permettent plus facilement que les autres formes d'entreprise ou d'organisation de mobiliser une grande diversité de ressources (non marchandes et non monétaires) qui s'ajoutent aux ressources provenant de l'État (EVERS et LAVILLE, 2004; LAVILLE, 1994). Cette mobilisation de diverses ressources est favorisée sans doute par les formes institutionnelles que représentent les associations sans but lucratif et les coopératives sociales, mais aussi par le fait que les services d'aide à domicile sont des quasi biens collectifs. Si ces services sont destinés principalement à des individus en situation de dépendance, ils ont également des retombées sociales, notamment sur la cohésion sociale, les collectivités locales et l'ensemble des parties prenantes (LAVILLE et NYSSENS, 2001, p. 16 et 218).

Les études empiriques sur les services sociaux, qui ont été initiées par CIRIEC international en 1998 sous la coordination de Jacques Defourny et poursuivies par EMES, ont porté sur neuf pays avec dix-sept chercheurs, dans un premier temps (LAVILLE et NYSSENS, 2001), et sur six pays avec une douzaine de chercheurs, dans un deuxième temps (EVERS et LAVILLE, 2004). D'autres recherches empiriques méritent d'être signalées dont celle d'Enjolras (1995) portant sur quatre pays (France, Norvège, Royaume-Uni et ÉtatsUnis) et celle sous la direction de Vaillancourt, Aubry et Jetté (2003) qui porte exclusivement sur le Québec mais qui s'avère exemplaire en termes de dimensions d'analyse (rapports de consommation, rapport de travail, rapport de genre et rapport de territoire) et de mise en rapport avec les politiques publiques québécoises dans le domaine. Ces recherches mettent en lumière autant (sinon plus) le potentiel de transformation que le potentiel d'innovation des associations et coopératives dans le domaine de l'aide à domicile. En premier lieu, les associations, qui avaient joué un rôle de revendication sous l'État-providence, se transforment de plus en plus en prestataires de services, modifiant par le fait même les rapports entre l'État, le marché, la société civile et le monde domestique (la famille). En deuxième lieu, les transformations de la régulation de ces rapports, qui sont sans doute tributaires des héritages et du chemin parcouru (path dependency) (ESPING-ANDERSEN, 2002 et 1999), laissent voir au moins trois formes de régimes en formation (idéal-types) mais souvent hybridées dans la réalisé, soit un régime libéral ou néo-libéral d'État-providence qui s'en remet au marché en conjonction ou en concurrence avec les associations sans but lucratif, un régime social-étatiste ou néoprovidentialiste où les associations et les coopératives sociales sont invitées à compléter l'offre étatique de services publics sans grande rupture avec la tradition tutélaire, un régime partenarial ou solidaire qui considérerait les associations et coopératives sociales comme des partenaires respectant ainsi leur autonomie et leur spécificité (VAILLANCOURT, AUBRY et JETTÉ, 2003, p. 289; ENJOLRAS, 1995, p. 238; EVERS et LAVILLE, 2004).

Le potentiel d'innovation des associations et coopératives dans le domaine de services sociaux serait variable selon que ces dernières participent à un régime d'État providence plus respectueux de leur autonomie et de leur spécificité (EVERS et LAVILLE, 2004). En revanche, l'étude de cas québécois a l'avantage de montrer que, pour un même régime, les associations et les entreprises peuvent elles-mêmes adopter une logique d'action qui les positionne dans une trajectoire d'innovation s'inscrivant dans l'un ou l'autre des trois régimes identifiés précédemment (VAILLANCOURT, AUBRY et JETTÉ, 2003). Autrement dit, dans un même cadre institutionnel, les initiatives relevant de l'économie sociale peuvent se donner un espace d'innovation plus ou moins restreint selon les choix stratégiques qu'ils adoptent à l'égard de l'État et du marché et selon l'importance qu'ils accordent à la démocratie participative, à la multiplicité des parties prenantes, les rapports aux usagers, aux travailleurs, aux femmes, aux territoires. Il apparaît également que les statuts juridiques adoptés par ces organisations (associations sans but lucratif, coopératives d'usagers, coopératives de travail et coopératives de solidarité) traduisent déjà des choix d'autant plus déterminants qu'ils sont plus rigides encore que les seuls choix stratégiques adoptés par la suite. Enfin, une conclusion qui résulte de cette recherche serait que «la mise en place du réseau des EESAD (entreprises d'économie sociale dans l'aide à domiciles) représentait une innovation sociale importante au moment 
de son institutionnalisation mais, contrairement à ce que nous pouvions espérer, les résultats au chapitre des rapports de consommation et des rapports de travail au sein de ces entreprises apparaissent bien en deçà des possibilités novatrices qu'offre théoriquement le type d'organisation de ces entreprises » (AUBRY, JETTÉ et VAILLANCOURT, 2004, p. 5). Si le financement insuffisant de la part des pouvoirs publics semble en grande partie responsable de ce résultat, il n'en demeure pas moins que d'autres études réalisées ailleurs arrivent à des conclusions similaires.

Suite aux recherches empiriques que nous avons examinées, il apparaît clairement qu'il serait hasardeux de vouloir rendre compte du potentiel d'innovation de l'économie sociale sans tenir compte du cadre institutionnel (micro et macro), des secteurs d'activités et des générations d'associations et de coopératives. Sous cet angle, nous devons prendre avec circonspection des recherches quantitatives comme celle réalisée par Osborne (1994) sur les organisations volontaires et non orientées vers le profit (VNPO) dans le domaine de la santé et des services sociaux en Angleterre. À partir d'une typologie des innovations qui tient compte du domaine particulier des services, l'auteur identifie trois types d'innovation, soit celles qui permettent : 1) d'atteindre de nouvelles clientèles (expansionary) ; 2) de proposer de nouveaux services (evolutinary) et 3 ) de faire les deux en même temps : nouvelles clientèles et nouveaux services (total). Comme sa définition des innovations met l'accent sur les discontinuités, il exclut les innovations incrémentielles, qui sont classées sous le registre du changement organisationnel (developmental). Cette exclusion se justifie selon lui par le fait que les « innovations incrémentielles » n'entraînent ni de nouveaux services, ni de nouvelles clientèles, mais que des améliorations sans grande rupture ou discontinuité. Au terme de son analyse, Osborne conclut sans doute avec trop de confiance que les VNPO sont faiblement innovatrices voire pas du tout :

Overall, this show innovative activity being reported by about a third of the respondents $(37,9 \%)$, and with almost half of them $(48,2 \%)$ reporting traditional activity alone. [...] On the basis of this initial evidence, it is therefore no longer possible to assert that VNPOs are innovative. As a whole, they are not. (OSBORNE, 1994, p. 1149).

Sans remettre en cause la méthodologie de cette recherche qui se considère comme la plus complète jamais réalisée sur les innovations dans les associations, nous devons reconnaître que la conceptualisation des innovations de l'auteur n'est pas complètement convaincante. Au départ, il avoue lui-même que sa typologie, y compris le terme $\mathrm{d}^{\prime}$ « incremental development ", est une adaptation au domaine des services de la typologie utilisée par Abertnathy, Clarke et Kantrow (1983) pour l'étude de la production industrielle. En deuxième lieu, il considère les associations dans la perspective d'un quasi marché où les innovations se limitent aux nouveaux services (services et processus tendant à se confondre) et aux nouvelles clientèles. Dès lors, les innovations sociales, que constituent les innovations organisationnelles et institutionnelles, sont considérées comme relevant du développement organisationnel, soit des améliorations incrémentielles qui n'entraînent pas de rupture ou de discontinuité dans les services et les clientèles. À notre avis, Osborne sous-estime grandement les innovations incrémentielles, mais sa recherche nous apprend tout de même quelque chose d'important, à savoir que l'économie sociale pourrait être plus particulièrement fertile en innovations incrémentielles en raison de la coopération entre les parties prenantes. Enfin, cette recherche néglige grandement le rapport des associations au regime d'État-providence du RoyaumeUni et plus largement aux transformations sociales.

\section{Conclusion}

Si l'économie sociale peut être considérée comme innovatrice, c'est parce qu'elle met en interaction, dès sa création, un regroupement de personnes et une entreprise ou une organisation capable de mobiliser des ressources financières et humaines pour produire des biens ou des services. Cette matrice d'innovation fait en sorte qu'elle pourra multiplier les innovations marginales ou incrémentielles qui reposent sur la coopération des parties 
prenantes. Dans certains domaines, comme celui de l'insertion ou de l'entrée des syndicats dans le capital de risque, on y retrouvera également des innovations radicales. Par ailleurs, les recherches empiriques révèlent aussi que le potentiel d'innovation des entreprises d'économie sociale n'est pas pleinement actualisé pour diverses raisons, dont celles provenant de l'environnement, à commencer par le mode de régulation et le financement accordé par l’État.

Par ailleurs, même si le terme «d'innovation » est souvent utilisé dans les recherches portant sur l'économie sociale, les chercheurs ont très peu systématisé et spécifié cette notion. De plus, ils semblent avoir ignoré complètement les apports théoriques réalisés principalement par les économistes hétérodoxes, à partir principalement des innovations technologiques et scientifiques, surtout dans le développement économique (LÉVESQUE, 2005). Cette littérature pourrait permettre de mieux comprendre non seulement la complexité et la diversité des innovations, mais également leur rapport aux transformations sociales. En ce qui concerne les innovations comme telles, nous avons déjà mentionné la distinction entre innovations radicales et innovations incrémentielles, ces dernières étant apparemment plus fréquentes dans les pays et les entreprises ouvertes à la coopération (HALL et SOSKICE, 2001). De même, le fait que les innovations résultent de processus sociaux nous invite à tenir compte de la durée alors que le fait qu'elles soient favorisées ou non par l'environnement social et institutionnel suggère la pertinence de la notion de système social $\mathrm{d}$ 'innovation pour rendre compte entre autres de la spécificité de certains secteurs et de certaines régions. Ainsi, les trois dernières décennies ont révélé l'émergence de nouveaux systèmes d'innovation en économie sociale, où l'on a vu apparaître des ressources techniques et professionnelles (ex. GRT, CDR, CDÉC) de même que des fonds dédiés à l'économie sociale, sans oublier des réseaux de recherche plus spécialisés. Plusieurs de ces nouveaux éléments relèvent eux-mêmes de l'économie sociale, même si leur émergence ne fut possible qu'avec le soutien des pouvoirs publics. Parmi les autres notions, qui pourraient enrichir les analyses des innovations, nous avons identifié celles de paradigme social, celle de sentier ou de trajectoire d'innovation et plus largement de modèle de développement et de régime de gouvernance.

Les trois dernières décennies (milieu des années 1970 à nos jours) ont vu naître une nouvelle génération d'associations et de coopératives dans le développement économique et dans le développement social, mais souvent à la frontière des deux. Comme les générations precedentes (DEMOUSTIER, 2001, p. 20-50), cette nouvelle génération a donné lieu à plusieurs grappes d'innovations sociales, sans doute de nouveaux services et de nouvelles clientèles mais aussi des innovations institutionnelles et organisationnelles. Ces innovations prennent toute leur signification non seulement au regard de la crise économique et sociale mais aussi des transformations sociales en cours (socio-économiques et sociopolitiques). Dans un premier temps, les nouvelles initiatives de la société civile ont pu être interprétées comme de simples réponses à des urgences devant une nouvelle question sociale, celle de l'exclusion et d'une nouvelle pauvreté résultant d'une société et d'une économie transition. De même, la préoccupation voire l'attention pour les innovations fut parfois interprétée comme un repli au regard des perspectives apparemment plus larges qu' offraient les entreprises alternatives de la fin des années 1960. Dans un deuxième temps, soit notamment à partir des années 1990, ces initiatives relevant de l'économie sociale sont de plus en plus analysées comme participant à des transformations sociales touchant la régulation, la reconfiguration de l'Étatprovidence et plus largement le " modèle de développement ", soit les rapports entre le développement économique et le développement social (ce qui implique une redéfinition de ce qu'il faut entendre par économie et social), sans oublier la ré-articulation entre le national, le mondial et le local (LÉVESQUE, BOURQUE et FORGUE, 2001).

Sous l'angle des transformations de l'État et de la régulation, il semble bien que l'économie sociale a contribué à l'émergence d'un nouveau paradigme social, une nouvelle vision des problèmes sociaux et des solutions à y apporter, de nouveaux principes tels ceux de la solidarité et de l'équité plutôt que de l'égalité, du ciblage des interventions plutôt que de l'universalité et $\mathrm{du}$ « mur à mur », le développement de ressources humaines, la responsabilisation et des 
mesures actives d'emploi, plutôt que l'assistance et des mesures passives, des investissements sociaux, plutôt que des dépenses sociales orientées vers la seule réparation (LÉVESQUE, 2003; NOËL, 1996).

Elle a également contribué à des expérimentations et à la mise au point de mécanismes et de façons de faire qui se sont maintenant imposés, comme c'est le cas avec l'insertion. Plus largement, l'économie sociale a probablement joué un rôle déterminant dans la redistribution de la place respective de l'État, du marché et de la société civile dans le domaine des services sociaux, notamment au niveau des prestations. Comme on a pu le voir avec les recherches examinées, les nouveaux arrangements institutionnels sont par ailleurs contrastés selon justement la reconnaissance et l'autonomie qu'ils accordent à l'économie sociale ou aux associations. Les formes qui apparaissent à première vue les plus ouvertes à la participation et à une certaine démocratisation, seraient celles qui font de l'économie sociale un partenaire à part entière et non pas un sous-traitant. Les générations précédentes de l'économie sociale sont également parties prenantes de ces transformations, mais elles se doivent d'opérer des choix stratégiques conséquents pour jouer un rôle comparable à celui de la nouvelle génération, soit des choix stratégique allant dans le sens de la focalisation, soit d'un revitalisation de l'association alors que s'opèrent des repositionnements sur le marché (MALO et VÉZINA, 2004), ce qui ne va pas de soi puisque la tendance «naturelle » des entreprises matures serait de concurrencer l'entreprise capitaliste sur son propre terrain (tendance également présente pour les associations des générations précédentes qui se sont souvent rapprochées des formes étatiques, à travers l'isomorphisme institutionnel).

À coup sûr, les trois dernières décennies ont été particulièrement fertiles en innovations de toutes sortes, mais également d'innovations sociales. Cette période tire-telle à sa fin? Il est plus que probable qu'à la suite de son institutionnalisation la dernière génération d'économie sociale entrera dans une nouvelle phase de développement où son potentiel d'innovation sociale risque d'être plus réduit. Dans ce cas, les innovations mises de l'avant par l'économie sociale cessent d'être un "fait social nouveau » puisqu'elles participent " dorénavant à ce qui fonde l'activité sociale d'une collectivité donnée » (NOREAU, 2003, p. 102). Toutefois, cette institutionnalisation lorsqu' elle se produit, révèle que les innovations sociales ont par ailleurs participé à des transformations sociales significatives. De plus, lorsque cette transformation atteint une forme relativement stabilisée, on peut supposer que les chercheurs intéressés jusque-là par ces entreprises ou organisations se désintéresseront d'autant plus qu'elles se seront banalisées voire standardisées. C'est ce que suggérait Perri 6 en 1994 (PERRI 6, 1994, p. 406) lorsqu'il écrivait que les chercheurs se désintéresseront de ce domaine de recherche, dans une décennie ou plus. Pour notre part, nous aurions tendance à questionner cette hypothèse, non pas à partir des effets de la standardisation que produit trop souvent l'institutionnalisation, mais sur la base des caractéristiques du nouveau modèle de développement et de son besoin d'innovation continue.

En effet, plusieurs études réalisées à partir de bases théoriques passablement différentes et parfois même contradictoires mettent de l'avant une économie et une société reposant sur l'innovation continue. Sans entrer dans les controverses, relevons que les innovations continues et quasi permanentes résulteraient entre autres

- D'une économie de service, une économie relationnelle, une économie anthropogénétique (une économie de production de personnes par d'autres hommes comme en témoigne l'importance croissante de la santé et de l'éducation). Selon une étude de l'OCDE (2000), qui laisse bien voir le poids de cette nouvelle économique, « les Américains dépensent plus pour leur santé qu'ils n'achètent de biens durables » (OCDE, 2000) 1960: biens durables $(14 \%)$, santé (5\%) 2000: biens durables (12\%), santé $(15,7 \%)$ ) (BOYER, 2002, p. 184).

- D'une économie devenue " économie cognitive » où l'information, le savoir et la connaissance occupent de plus en plus d'espace, une économie qui prend sa source dans 
l'organisation ellemême et « dans le processus cognitifs interactifs de codification à travers la numérisation des savoirs jusque-là tacite et leur captation aussi bien par l'entreprise que par le marché et la puissance publique » (MOULIER BOUTANG, 2003, p. 307).

- D'une économie qui reposerait de plus en plus sur une croissance endogène échappant ainsi à la logique des rendements décroissants, au moins partiellement ou pour un temps (même si la théorie de la croissance endogène est controversée, elle attire tout de même l'attention sur des nouveaux facteurs de production que sont les connaissances, des facteurs non marchands et extra-économiques).

- De la mondialisation et des nouvelles technologies de communication qui font que les meilleurs sont en concurrence directe avec les meilleurs à l'échelle du monde et que ce sont généralement ceux qui sont les plus innovateurs qui s'imposent.

En somme, le très grand intérêt suscité par les innovations au cours des dernières décennies résulterait non seulement du fait que nous sommes progressivement passés d'un modèle de développement à dominante manufacturière à un autre modèle, mais aussi du fait que cet autre modèle de développement serait basé sur la connaissance et les services relationnels, rendant ainsi possible l'innovation continue et quasi-permanente, tendance soutenues par les nouvelles technologie et exacerbée par la mondialisation. Si cette hypothèse de l'innovation continue devait se vérifier, il faudrait ajouter que les innovations sociales occuperont un espace de plus en plus grand en raison non seulement des perturbations engendrées par les innovations continues mais aussi de la mobilisation plus intensive et plus large de ressources en grande partie non marchandes qu'elles exigent. Ce que Chambon, Alix et Devevey $(1982$, p. 5) avançaient au début des années 1980 nous semble encore plus vrai aujourd'hui qu'il y a plus de vingt ans : « on a affaire, écrivaient-ils, à des civilisations où les problèmes de sociétés civiles se résolvent naturellement par des pratiques du type innovations sociales».

\section{Notas}

${ }^{1} 1$ Comme la plupart des recherches tendent à le montrer (DEFOURNY et MONZON, 1992; DESROCHE, 1983; DRAPERI, 2000; DEMOUSTIER, 2001; GUESLIN, 1998; LAVILLE, 1994; VIENNEY, 1994), la résurgence de l'économie sociale comme référence explicite traduit quelque chose de nouveau, soit la redécouverte d'une parenté, qui existait sans doute auparavant, mais qui était occultée par un repli sur sa propre composante ou famille, en l'occurrence coopérative, mutualiste ou associative. Cette redécouverte ne saurait s'accompagner de la perte des identités propres à chacune des composantes, mais elle prend une portée nouvelle donnant lieu à de nouvelles collaborations et coopérations. Autrement dit, l'idée de l'économie sociale permettrait non seulement d'établir des passerelles entre les diverses familles mais aussi de découvrir des raisons nouvelles de le faire. Dans le cas contraire, il faudrait conclure que cette notion est utile aux chercheurs pour mettre en lumière la parenté entre les composantes, mais n'ajoute rien de nouveau à ce que les diverses familles ainsi réunies apportent déjà et depuis longtemps.

${ }^{2}$ Nous avons défini ailleurs les innovations organisationnelles comme celles que l'on retrouve au niveau « des formes de la division et de la coordination du travail, des modes de gestion, des modalités de coordinations et des interactions sociales », soit « le lieu également des apprentissages collectifs et des formes de gouvernance ». Par la même occasion, nous avons défini les innovations institutionnelles comme celles que l'on retrouve au niveau « des systèmes de règles, de partage des droits et responsabilités, des systèmes de gestion des conflits, du système politique sans lequel aucune organisation et association ne fonctionneraient » (LÉVESQUE, 2004, p. 55-6).

${ }^{3}$ Elle ne l'est devenue que quelques mois avant sa disparition en 1982 pour avoir accès à un financement de la Société de développement coopératif. À l'occasion de cette demande appuyée par la SDC, le CCQ s'était montré neutre, ne formulant ni un avis positif, ni un avis négatif de sorte que la décision d'octroyer le statut coopératif à Tricofil fut prise par le Ministère en charge des coopératives et non par le CCQ (BOUCHER, 1982, p. 121 et 306).

${ }^{4}$ La loi des coopératives adoptées en 1984 permettra la formation de coopératives de travail dans des secteurs d'activités interdits jusquelà comme le commerce. Elle permettra également de former des coopératives de travail avec trois membres.

${ }^{5}$ Ainsi, en dépit d'un soutien significatif de la part des pouvoirs publics québécois, le nombre de coopératives de travail y est passé de 141 à 191 sur une période de dix ans, soit de 1993 à 2003, alors que le nombre de constitutions (créations) de nouvelles coopératives de travail) aurait été de 449 coopératives de travail pour une période comparable, celle de 1995 à 2004 (MDEIE, 2004). Enfin, de 1999 à 2003, le nombre total d'emplois, l'actif et le chiffre d'affaires de l'ensemble des coopératives de travail ont légèrement reculés.

${ }^{6}$ Les coopératives de travailleurs actionnaires (CTA) qui constituent une innovation spécifique au Québec, présentent quelques ressemblances avec les ESOP dans la mesure où elles réunissent les travailleurs qui investissent dans l'entreprise qui les emploie. Cependant, les investissements sont faits par le collectif de travailleurs, soit la CTA, 
de sorte que ces derniers ne sont pas dans un rapport individualisé avec l'entreprise mais dans un rapport collectif (LÉVESQUE, 1994). Entre 1993 et 2003, le nombre de CTA est passé de 24 à 61 (MDEIE, 2004, p. 44) alors que le nombre de constitutions (créations) a été de 186 (MDEIE, 2004, p. 16).

\section{Références}

ABERNATHY, W.; CLARKE, K.; KANTROW, A. Industrial Renaissance. New York: Basic Books, 1983.

AMABLE, Bruno; BARRÉ, Rémi; BOYER, Robert . Les systèmes d'innovation à l'ère de la globalisation. Paris: Économica, $1997,402 \mathrm{p}$.

AUBRY, François; JETTÉ, Christian; VAILLANCOURT, Yves .L'économie sociale dans les services à domicile: une source d'innovation sociale?. Montréal: Cahier du LAREPPS (no 04-31), 2004, 24 p.

AUCLAIR, René; Christiane LAMPRON. Approche intégrée: une innovation sociale dans la dispensation des services sociaux. Service Social, v. 36, n. 2/3, p. 315-41, 1987

BÉLANGER, Paul R.; LÉVESQUE, Benoît. La théorie de la régulation, du rapport salarial au rapport de consommation. Un point de vue sociologique. Cahiers de recherche sociologique, n. 17, p. 19-61, 1991.

BÉLANGER, Paul R.; GRANT Michel; LÉVESQUE, Benoît (sous la dir.). Modernisation des entreprises et relations de travail. Montréal: PUM, 1994, 320 p.

BÉLANGER, Paul R.; LAPOINTE, Paul-André; LÉVESQUE, Benoît. Les innovations organisationnelles et les blocages dans les entreprises. In: MURRAY, G.; BÉLANGER J. ; GILES, A.; LAPOINTE, P.-A. (sous la dir.). L'organisation de la production et du travail : vers un nouveau modèle?. Québec: Presses de l’Université Laval, 2004, p. 155-90.

BJÖRKMAN, Hans. Service Innovation. A Collaborative Approach, 2001. In: ADLER, Niclas; BORZAGA, Carlo; DEFOURNY, Jacques (sous la dir.). The Emergence of Social Enterprise. London et New York: Routledge, 2004, 382 p.

BORZAGA, Carlo; SPEAR, Roger (sous la dir.). Trends and Challenges for Co-operatives and Social Enterprises in developed and transition countries. Trento: Edizioni, 2004, 280 p.

BORZAGA, Carlo; GUI, Benedetto; POVINELLI, Fabrizio. 1998. Le rôle des entreprises d'insertion sur le marché du travail. L'éclairage d'une analyse économique. In: DEFOURNY, Jacques; FAVREAU, Louis; LAVILLE, Jean-Louis (sous la dir.). Insertion et nouvelle économie sociale. Paris: Desclée de Brouwer, p. 267-92.

BORZAGA, Carlo. Italie. L'impressionnant développement des coopératives sociales. 1998. In: DEFOURNY, Jacques; FAVREAU, Louis; LAVILLE, Jean-Louis (sous la dir.). Insertion et nouvelle économie sociale. Paris: Desclée de Brouwer, p.99-126.

BOUCHARD, Camil. Recherche en sciences humaines et sociales et innovations sociales. Contribution à une politique de l'immatériel. Québec: Groupe de travail sur l'innovation sociale, 1999, 26 p.

BOUCHER, Paul-André; MARTEL, Jean-Louis. Tricofil tel que vécu!. Montréal: Éditions des H.É.C., 1982, 380 p.

BOYER, Robert. Histoire des techniques et sciences sociales. Cahier de recherche sociologie, n. 17, p. 53-80, 1991.

. La croissance, début du siècle. De l'octet au gène. Paris: Albin Michel, 2002, 234 p.

BRACZYK, Hans-Joachim; COOKE, Philip; HEIDENREICH, Martin (dir.). Regional Innovation Systems. The role of governance in a globalized World. London et New York: Routledge, 1998, 499 p.

CALLON, Michel; LARÉDO Philippe; RABEHARISOA, Vololona . Que signifie innover dans les services. Une triple rupture avec le modèle de l'innovation industrielle. La Recherche, n. 295, février 1997, p. 34-6.

CALLON, Michel. La science et ses réseaux. Genèse et circulation des faits scientifiques. Paris: Éditions La Découverte, 1989.

CASSON, Mark. L'entrepreneur. Paris: Economica, 1982, 388 p.

CHAMBON, Jean-Louis; DAVID, Alix; DEVEVEY, Jean-Marie. Les innovations sociales. Paris: PUF, Collection Que sais-je?, 1982.

CHANTIER DE L'ÉCONOMIE SOCIALE. Pour un renforcement du partenariat au services des personnes éloignées ou exclues du marché du travail. Rapport synthèse du comitê de travail initié par le Chantier de l'économie sociale, en collaboration avec la Coalition des organismes communautaires pour le développement de la main-d'œuvre. Montréal, 2005, 59 p.

CIREC Espana. Rapport de synthèse sur l'Économie sociale en Espagne en 2000. Valence: Universitat de Valencia, 2002, 154 p. (Rapport commandité part le Ministrio de Trabajo y asuntos sociales et Direccion General de Fomento de la Economia social y des Fondo social Europeo).

CNLAMCA; CIRIEC. Économie sociale. Débat européen. Mutualité, coopération, association. Actes du pré-colloque de Bruxelles. Paris: CIEM, 1979, 94 p.

COLEMAN, James S. Social Invention. Social Forces, v. 42, n. 2, p. 163-73, 1970.

COMEAU, Yvan; LÉVESQUE, Benoît. Workers' Financial Participation in the Property of Enterprises in Québec. Economic and Industrial Democracy, v. 14, n. 2, p. 233-50, 1993. 
COMEAU, Yvan; FAVREAU, Louis; LÉVESQUE, Benoît; MENDELL, Marguerite. Emploi, économie sociale, développement local: les nouvelles filières, Sainte-Foy, Presses de l'Université du Québec, 2001, 302 p.

COMEAU, Yvan. Les coopératives de travail au Québec : un bilan de la situation. Coopératives et Développement, v. 23, n. 3, p. 59-76, 1992.

. Les éléments de satisfaction et d'insatisfaction dans les coopératives de travail au Québec. Coopératives et Développement, v. 25, n. 1, p.31-46, 1993.

. Grille de collecte et de caractérisation des données pour l'étude d'activités de l'économie sociale. Montréal: Cahier du CRISES, Collection Études théoriques, n. ET9605, 1996, 19 p.

CORIAT, Benjamin et Olivier WEINSTEIN. Les nouvelles théories de l'entreprise. Paris: Librairie générale française (Livre de poche), 1995, $220 \mathrm{p}$.

CÔTÉ, Daniel (sous la dir.). Les holdings coopératifs: évolution ou transformation définitive?. Bruxelles: De Boeck Université, 2000, 413 p.

CÔTÉ, Daniel. Cohésion coopérative et fonctionnement démocratique : clé de la gestion d'une grande entreprise. Économie et solidarités, v. 34, n. 2, 2003, p. 47-65.

CRADDOCK, Trent; KENNEDY, Sarah. Analyse des tendances internationales dans le domaine des coopératives de travail. Ottawa, Secrétariat des coopératives. Disponible en:<http://www.agr.gc.ca/policy/coop/analysis_f.phtml>. 2005

CST (Conseil de la science et de la technologie). L'innovation dans les services. Pour une stratégie de l'immatériel. Avis, Québec: CST, 2003, 118 p.

DACHEUX, Éric et Jean-Louis LAVILLE (dir.). Économie solidaire et démocratie. Hermès, n. 36, 250 p., 2004.

DEFOURNY, Jacques; FAVREAU, Louis; LAVILLE, Jean-Louis (sous la dir.). Insertion et nouvelle économie sociale, Paris: Desclée de Brouwer, 369 p., 1998.

DEFOURNY, Jacques; NYSSENS, Marthe; SIMON, Michel. Belgique. De l'association sans but lucratif à la société à finalité sociale. In: DEFOURNY, Jacques, FAVREAU Louis; LAVILLE Jean-Louis (sous la dir.), Insertion et nouvelle économie sociale, Paris, Desclée de Brouwer, 1998, p. 73-98.

DEFOURNY, Jacques, Michel SIMON et Sophie ADAM. 2002. Les coopératives en Belgique : un mouvement d'avenir?, Bruxelles, Éditions Luc Pire, 118 p.

. Démocratie coopérative et efficacité économique: 1'efficacité comparée des scop françaises. Bruxelles: De Boeck Université, 1990, 234 p.

. Le secteur de l'économie sociale en Belgique. In: DEFOURNY, J.; MONZON CAMPOS, J.-L. (dir.). Économie sociale, entre économie capitaliste et économie publique. Bruxelles: CIRIEC et de Boeck Université, 1992, p. 225-56.

Coopérative de travail : une recherche foisonnante et novatrice. Une interview de Jacques Defourny. Propos recueillis par Éric Bidet. Revue des études coopératives, mutualistes et associatives, n. 253-54, p. 23-35, 1994.

. Resocialiser l'économie ?. Revue internationale de l'économie sociale, n. 275-6, p. 184-192, 2000.

DELORS, Jacques. The European Union and the Third Sector. In: EVERS, Adalbert; LAVILLE , Jean-Louis (sous la dir.). 2004. EVERS, Adalbert; LAVILLE, Jean-Louis (dir). 2004. The Third Sector in Europe. Globalisation and Welfare. Cheltenham: Edward Elgar, 2004, p. 206-15.

DEMOUSTIER, Danièle. Analyse de l'emploi. 2000. In: CIRIEC. Les entreprises et organisations du troisième secteur. Un enjeu stratégique pour l'emploi. Liège: CIRIEC, 2000, p. 33-50.

. Les organisations d'économie sociale, acteur de la régulation socio-économique. Revue internationale de l'économie sociale. Paris, n. 275-6, p. 137-48, 2000.

. Analyse de l'emploi. In: CIRIEC. 2000. Les entreprises et organisations du troisième secteur. Un enjeu stratégique pour l'emploi, Liège, CIRIEC, 2000a, p. 3350.

2001. L'économie sociale et solidaire. S'associer pour entreprendre autrement. Paris: Syros, 208 p.

DESROCHE, Henri. Pour un traité d'économie sociale, Paris, Coopérative d'édition et d'information mutualiste, 1983, $254 \mathrm{p}$.

Histoires d'économies sociales. D'un tiers état aux tiers secteurs, 1791-1991. Paris: Syros, 1991, 262 p.

EME, Bernard; LAVILLE, Jean-Louis. Les petits boulots en question. Paris: Syros, 1988, 232 p.

EME, Bernard. Participation sociale et formes plurielles d'insertion. In: DEFOURNY, Jacques; FAVREAU, Louis; LAVILLE, Jean-Louis (sous la dir.). Insertion et nouvelle économie sociale. Paris: Desclée de Brouwer, 1998, p. $293-320$.

ENJOLRAS, Bernard. Vers une théorie socio-économique de l'association: l'apport de la théorie des conventions. In: Revue des études coopératives, associatives et mutualistes, n. 48, 1994, p. 93-106.

. Le marché providence. Aide à domicile, politique sociale et création d'emploi. Paris: Desclée de Brouwer, 1995, $254 \mathrm{p}$.

ESPING-ANDERSEN, Goasta (dir.). Why We Need a New Welfare State. Oxford: Oxford University Press, 2002. 
ESPING-ANDERSEN, Goasta. Les trois mondes de l'État-providence. Essai sur le capitalisme moderne. Paris, PUF, 1999.

EVERS, Adalbert et Jean-Louis LAVILLE (dir). The Third Sector in Europe. Globalisation and Welfare. Cheltenham: Edward Elgar, 2004, 266 p.

EVERS, Adalbert. Part of the Welfare Mix : The Third Sector as an intermediate area. Voluntas, v. 6, n. 2, p. 119-39, 1995.

FAGERBERG, Jan. Innovation: A Guide to Litterature, Oslo, Centre for Technology Innovation and Culture (University of Oslo) (Article présenté à l'atelier, The Many Guides of Innovation: What we have learnt and where we are heading. Ottawa, 23-24 octobre 2002, Statistiques Canada. Également publié dans Handbook of Innovation, Oxford), 2003.

FAVREAU, Louis; LAROSE, Gérald; FALL, Abdou Salam. (dir.), Altermondialisation, économieet coopération internationale. Québec et Paris: Presses de l’Université du Québec et Karthala, 2004, 386 p.

FAVREAU, Louis. Québec. L'insertion conjuguée avec le développement économique communautaire. In: DEFOURNY, Jacques; FAVREAU, Louis; LAVILLE, Jean-Louis (sous la dir.). Insertion et nouvelle économie sociale. Paris: Desclée de Brouwer, 1998, p. 159-82.

FECHER, Fabienne; LÉVESQUE, Benoît. Avec la collaboration de Philippe GROSJEAN, François-Louis THOREAU et Christine DUSSART. Cadrage de l'économie sociale en Région wallonne. Rapport Final. Liège: CIRIEC, 2005, 119 p.

FECHER, Fabienne et al. L'économie sociale d'insertion. Analyse des complémentarités et de l'efficacité des outils d'économie sociale. Gent: Academia Press, 2002, 306 p.

FERREIRA, Nathalie. Économie sociale et autogestion: entre utopie et réalité. Paris: L'Harmattan, 2004, 240 p.

FONTAN, Jean-Marc; KLEIN, Juan-Luis; LÉVESQUE Benoît (dir). Reconersion économique et développement territorial. Le rôle de la société civile. Québec: Presses de l'Université du Québec, 2003, 340 p.

FRASER, Mario. Les organisations oeuvrant dans le domaine de l'innovation sociale: résultat d'une recherche dans l'Internet. Québec: CST, 2003, 39 p.

FREEMAN, Christopher. The Economics of Industrial Innovation. 2nd édition. 1986. Francis Pinter, London, 1982, 256 p. FREEMAN, Christopher. Innovation, Change of Techno-Economic Paradigm and Biological Analogies in Economics. Revue Économique, n. 2, p. 211-31, 1991.

GABORD, Dennis. Innovations: scientific, technological and social. London: Oxford University Press, 1970, 109 p.

GIRARD, Jean-Pierre. Solidarity Co-operatives fin Quebec (Canada): overview, 2004. In: BORZAGA, Carlo; SPEAR, Roger (sous la dir.). Trends and challenges for co-operatives and social enterprises in developed and transition countries. Trento: Edizioni, 2004, p. 165-82.

GOMEZ, Pierre-Yves. Le gouvernement de l'entreprise: modèles économiques et pratiques de gestion. Paris: InterEditions, 1996, $271 \mathrm{p}$.

GOUVERNEMENT DU QUÉBEC. Politique québécoise de la science et de l'innovation. Savoir changer le monde. Québec: Ministère de la science et de la technologie, 2001, 170 p.

GUESLIN, André. L'invention de l'économie sociale. Paris: Economica, 1998, 430 p.

HALL, David J. Social relations and innovation. London: Routledge and Kegan Paul Ltd, 1977.

HALL, Peter et David SOSKICE. Varieties of Capitalism: the Institutional Foundations of Comparative Advantage. Oxford: Oxford University Press, 2001. Traduction du texte de Hall et Soskice. Les variétés du capitalisme. L'année de la régulation. v. 6. Paris: Presses de Sciences-Po, p. 47-123, 2002.

IGNATIEFF, Nicolas A.; MALO, Marie-Claire . Caisses Desjardins et cercles d'emprunt à Montréal: quelle configuration partenariale?. Économie et Solidarités, v. 29, n. 1, p. 81-90, 1997.

JEANTET, Thierry; VERDIER, Roger . L'économie sociale. Paris: CIEM, 1982, 578 p.

JETTÉ, Christian; LÉVESQUE, Benoît; MAGER , Lucie; VAILLANCOURT, Yves. Économie sociale et transformation de l'État-providence dans le domaine de la santé et du bien-être. Une recension des écrits. 1990-2000. Québec: Presses de l'Université du Québec, 2000, 202 p.

JOYAL, André. Les entreprises alternatives au Québec. In: LÉVESQUE, B.; CHOUINARD, O.; JOYAL, A. (sous la dir.). L'autre économie, une économie alternative?. Québec: Presses de l'Université du Québec, 1989, p. 165-84.

LAROCQUE, Paul. Un mouvement centrifuge: la récente transition des pêcheries maritiques au Québec. In: Coopératives et Développement, v. 19, n. 2, 1988, p. 15-27.

LATOUR, Bruno. lère édition: 1989, La science en action. Paris: Gallimard, 1995, 663 p. (une préface a été ajoutée à l'édition de 1995).

LAVILLE, J.-L. et al. Tiers système : une définition européenne. In: CIRIEC. 2000. Les entreprises et Organisations du troisième secteur. Un enjeu stratégique pour l'emploi. Liège: CIRIEC, 2000, p. 107-29.

LAVILLE, Jean-Louis; NYSSENS, Marthe (sous la dir.). Les services sociaux entre association, État et marché. L'aide aux personnes âgées. Paris: La Découverte/Mauss/CRIDA, 2001, 286 p. 
LAVILLE, Jean-Louis; LÉVESQUE, Benoît; MENDELL, Marguerite. The Social Economy. Diverse approaches and practices in Europe and in Canada, OCDE/LEED. 2005. The Social Economy as a Tool of Social Innovation and Local Development, Paris, OCDE/LEEE, 2005, p. 125-73.

LAVILLE, Jean-Louis. Les coopératives de travail en Europe pour un bilan 1970-1980. Coopératives et Développement, v. 25, n. 1, p. 5-29, 1993.

. L'économie solidaire. Une perspective internationale. Paris: Desclée de Brouwer, 1994, 334 p.

. L'économie sociale revisitée à la lumière de l'insertion. In: DEFOURNY, Jacques; FAVREAU, Louis; LAVILLE, Jean-Louis (sous la dir.). Insertion et nouvelle économie sociale. Paris: Desclée de Brouwer, 1998, p. 321-36.

. Sociologie des services. Entre marché et solidarité. Ramonville Saint-Agne: Érès, 2005, 177 p.

LEPAGE, Henri. L'autogestion. Dossier préparatoire mis à l'étude par l'Institut de l'entreprise. Paris: Institut de l'entreprise, 1976, 59 p. Disponible à: <http://www.institut-entreprise.fr/index.php?id=534>.

Autogestion et capitalisme. Paris: Institut de l'Entreprise-Masson, 1978, 450 p.

LÉVESQUE, Benoît; CÔTÉ, Daniel . Le changement des principes coopératifs à l'heure de la mondialisation: à la recherche d'une méthodologie. In: Alberto ZEVI; CAMPOS, José Luis Monxon (sous la dir.). Coopératives, marchés, principes coopératifs. Bruxelles: CIRIEC et De Boeck Université, 1995, p. 1-16.

LÉVESQUE, Benoît; MENDELL, Margie. L'économie sociale: diversité des approches et des pratiques. Conseil de recherches en sciences humaines du Canada, 2 juillet 2004. Disponible à: <http://www.sshrc.ca/web/apply/ background/social_economy_f.pdf> (publié avec de légères modifications sous le titre: The Social Economy: Diverse Approaches and Practices. Journal of Rural Cooperation, v. 33 n. 1, 2004).

LÉVESQUE, Benoît; MENDELL, Marguerite. Les fonds régionaux et locaux de développement au Québec: des institutions financières relevant principalement de l'économie sociale. In: Marc-Urbain PROULX (dir.), Territoire et développement économique. Paris et Montréal: L'Harmattan, 1998, p. 220-70.

LÉVESQUE, Benoît; MALO, Marie-Claire. Les études sur les coopératives: base de données bibliographiques, centres de recherche universitaires. In: SÉGUIN, Marie-Thérèse (sous la dir.). Pratiques coopératives et mutations sociales. Paris: L'Harmattan, 1995, p. 247-67.

Un nouveau Desjardins à l'ère de la globalisation: législation et pratiques coopératives dans les caisses d'épargne et de crédit. In: ZEVI, Alberto; MONZON, José Luis (sous la dir.). Coopératives, marchés, príncipes coopératifs. Bruxelles: De Boeck, 1995a, p. 243-77.

LÉVESQUE, Benoît; CÔTÉ, Alain; CHOUINARD, Omer; RUSSELL, Jean-Louis. Profil socio-économique des coopératives de travail au Québec. Montréal: UQAM et Comitê provincial de coopératives production, de travail et de précoopératives, $1985,180 \mathrm{p}$.

LÉVESQUE, Benoît; BOURQUE, Gilles; FORGUES, Éric. La nouvelle sociologie économique. Originalité et tendances nouvelles. Paris: Desclées de Brouwer, 2001, 268 p.

LÉVESQUE, Benoît; BÉLANGER, Paul R ; BOUCHARD, Marie; MENDELL, Marguerite; LAVOIE, André; GENDRON, Corinne; ROUZIER, Ralph. Le Fonds de solidarité (FTQ). Un cas exemplaire de nouvelle gouvernance. Montréal: Fonds de solidarité, 2000, 108 p.

LÉVESQUE, Benoît; BÉLANGER, Paul R.; MAGER, Lucie avec la coll. de D. MARTEL, Y. COMEAU, M.-C. MALO et alii. La réingénierie des caisses populaires et d'économie Desjardins. Contexte et études de cas, Montréal, CRISES/SAC/ FC-CSN, 1997, 298 p.

LÉVESQUE, Benoît; BÉLANGER, Paul R.; MAGER, Lucie. La réingénierie des services financiers: un secteur exemplaire de l'économie des services. Le cas des Caisses populaires et d'économie Desjardins. In: Lien social et Politiques - RIAC, n. 40, p. 89-103, 1999.

LÉVESQUE, Benoît. Une forme originale d'association capital-travail, les coopératives de travailleurs au Québec. In: Revue des études coopératives, mutualistes et associatives (Paris), n. 251, p. 49-60, 1994.

. Entrepreneurship collectif et économie sociale: entreprendre autrement, Montréal. Montréal: Cahier de l'ARUCÉS (I-02-2002), 2002, 35 p.

. Fonction de base et nouveau rôle des pouvoirs publics: vers un nouveau paradigme de l'État. Annals of Public and Cooperative Economics. Oxford (Blackwell), v. 74, n. 4, p. 489-513, 2003.

. Les entreprises d'économie sociale, plus porteuses d'innovations sociale que les autres?. In: Le développement social au rythme de l'innovation. Québec: Presses de l'Université du Québec et Fonds de recherche sur la société et la culture, 2004, p. 51-72.

Innovations et transformations sociales dans le développement économique et le développement social: approches théoriques et politiques publiques. Montréal: Cahiers du CRISES; Collection Études théoriques, n. ET0507, 80 p., 2005.

LIPIETZ, Alain. Choisir l'audace. Une alternative pour le XXIe siècle. Paris: La Découverte, 1989, 156 p.

LUNDVALL, Bengt-Ake (dir.). National System of Innovation. Toward a Theory of Innovation and Interactive Learning. 
London et New York, 1992, 342 p.

MAC PHERSON, Ian. Remembering the Big Picture: the Co-operative Mouvement and Contemporary Communities, 2004. In: BORZAGA, Carlo et Roger SPEAR (sous la dir.). Trends and challenges for co-operatives and social enterprises in developed and transition countries. Trento: Edizioni, 2004, p. 39-48.

MALO, Marie-Claire; VÉZINA, Martine. Gouvernance et gestion de l'entreprise collective d'usagers: stratégie de création de valeur et configuration organisationnelle. Économie et Solidarités, v. 35, n. 1, p. 100-20, 2004.

MALO, Marie-Claire; LÉVESQUE, B.; CHOUINARD, O.; DESJARDINS, P.-M.; FORGUES, E. Coopératives financières, cohésions sociale et nouveau territoire local à l'ère de la mondialisation. Montréal: Cahiers du CRISES, 2001, 65 p.

MALO, Marie-Claire. Le Mouvement Desjardins: modèle d'une économie solidaire?.Économie et Solidarités, v. 29, n. 1, p. 29-48, 1997.

. La gestion stratégique de la coopérative et de l'association d'économie sociale. 2e partie : L'entreprise et ses orientations. Revue internationale de l'économie sociale (RECMA), n. 282, p.84-94, 2001.

La gestion stratégique de la coopérative et de l'association d'économie sociale. 1e partie: L'entrepreneur et son environnement. Revue internationale de l'économie sociale (RECMA), n. 281, p.84-96, 2001a.

MINISTÈRE DÉVELOPPEMENT ÉCONOMIQUE, INNOVATION ET EXPORTATION (MDEIE). Coopératives du Québec données statistiques (édition 2005). Québec: MDEIE, 2005, 60 p.

MOSS, Thomas H. Social Invention and Innovation as partners in technological progress. In: LUNDSTEDT, Sven B.; COLGLAZIER, E. William (dir.). Managing Innovation: the social dimension of creativity, invention and technology. New York: Pergamon Press, 1982.

MOULIER BOUTANG, Yann. Capitalisme cognitif et nouvelles formes de codification du rapport salarial. In: VERCELLONE, Carlo (dir.). Sommes-nous sortis du capitalisme industriel ?. Paris: La Dispute, 2003, p. 305-20.

MUNKNER, Hans. Multi-stakeholder Co-operatives and their Legal Framework, 2004. In: BORZAGA, Carlo; SPEAR, Roger (sous la dir.). Trends and challenges for co-operatives and social enterprises in developed and transition countries. Trento: Edizioni, 2004, p. 49-82.

NELSON, R.R.; WINTER, S.G. National Systems of Innovation: A Comparative Study. Oxford: University Press, 1982. NOËL, A. Vers un nouvel État-providence? Enjeux démocratiques. Politique et Sociétés, v. 15, n. 30, p.1-28, 1996.

NOREAU, Pierre. L'innovation sociale et le droit. Est-ce bien compatible?. In: Le développement social au rythme de l'innovation. Québec: Presses de l'Université du Québec et Fonds de recherche sur la société et la culture, 2003, p. 73108.

OECD. Oslo Manual. Guidelines for Collecting and Interpreting Innovation Data. Paris: Third Edition, 2005, 162 p. OSBORNE, Stephen. Naming the Beast: Defining and Clarifying Service Innovations in Social Policy. Human Relations 9, v. 51, p. 1133-54, 1994.

Voluntary organization in the management of voluntary ande non-profit organization. London and New York, Routledge, 1994a.

PERRI 6; VIDAL, Isabel (sous la dir.). Delivering Welfare, repositioning non-profit and co-operative action in western European Welfare States. Barcelone: Centre d'iniciatives de l'Economia Social, 1994, 410 p.

PERRI, 6. Innovation by non-profit organizations: Policy and research issues. In: Non-Profit Management and Leadership, v. 3, n. 4, p. 397-414, 1993.

PESTOFF, Victor. 1995. Between Markets and Politics. Co-operatives in Sweden, Frankfurt AM Main. New York and Boulder: Campus Verlag and Westview Press, 1993.

PETIT, Pascal; SOETE, Luc. Progrès technique et nouveaux dualismes. In: Carlo Vercellone (dir.), Sommes-nous sortis du capitalisme industriel?. Paris: La Dispute, 2003, p. 91-120.

ROCARD, Michel. L'économie sociale et solidaire: une perspective Nord-Sud, discours de clôture. Revue internationale de l'économie sociale, n. 282, p. 6-16, 2001.

SALAMON, L. M.; ANHEIER, H. K. The Emerging Sector: An Overview. Baltimore: The Johns Hopkins University Institute for Policy Studies, 1994.

SCHUMPETER, Joseph A. Business Cycles: A Theoretical, Historical and Statistical Analysis of Capitalist Process. New York and London: Mc Graw-Hill, 2 v., 1939.

SHANI, A.B. Rami; STYHRE, Alexander (dir.), Collaborative Research in Organization. Foundations for Learning Change, and Theoretical Development. Sage Publications, p. 265-84.

SPEAR, Roger. From co-opertive to social enterprise: trends in European experience, 2004. In: BORZAGA, Carlo; SPEAR, Roger (sous la dir.). Trends and Challenges for Co-operatives and Social Enterprises in developed and transition countries. Trento: Edizioni, 2004, p. 99-116.

TAYLOR, James B. Improducting Social Innovation, Journal of Applied Behavioral Science, v. 6, n. 1, 1970, p. 69-77.

THÉRÊT, Bruno. Institution et institutionnalisme, vers une convergence des conceptions de l'institution?, 2000. In: 
TALLARD, Michèle; THÉRÊT, Bruno; URI, Didier. Innovations institutionnelles et territoire.Paris: L'Harmattan, 2000, p. 25-68.

TOURAINE, Alain. Un nouveau paradigme. Pour comprendre le monde d'aujourd'hui. Paris: Fayard, 2005, 366 p.

TREMBLAY, Benoît et Daniel Côté. Cooperative Banks and Mobilization of Capittal: To what End, with which Partners and with which consequences for Members? International Co-operative Banking Association Journal, n. 13, p. 5-23, 2001.

VÉZINA, Martine (sous la dir.). Grands groupes coopératifs dans le monde, in Économie et Solidarités, v. 34, n. 2, 2003a.

VÉZINA, Martine. Les grandes coopératives et leur institutionnalisation. Économie et Solidarités, v. 34, n. 2, p. 20-26, 2003.

VIENNEY, Claude. Les activités, les acteurs et les règles des organisations de l'économie sociale, DESup. Économie sociale, Octobre 1986. Université de Paris I. Centre d'éducation permanente. U.E.R. Travail et Études Sociales, 1986. . L'économie sociale. Paris: La Découverte, 1994, 125 p.

WEBER, Max. L'éthique protestante et l'esprit du capitalisme. Paris: Plon, 1964, 342 p.

WHYTE, William Foote. Social invention for solving human problems. American Sociological Review, v. 47, p. 1-13, 1982.

ZEVI, Alberto et José Luis MONZON CAMPOS (sous la dir.). Coopératives, marchés, principes coopératifs. Bruxelles: CIRIEC et De Boeck Université, 1996, 344 p.

ZIMMERMANN, Horst. Innovation in Non Profit Organizations. Annals of Public and Cooperative Economics (Oxford), v. 70, n. 4, p. 589-619, 1999. 